\begin{tabular}{ll}
\hline PSS & PROCEEDINGS \\
\hline
\end{tabular}

\title{
Muon cooling: a Higgs Factory at CERN ?
}

\section{Carlo Rubbia ${ }^{1}$}

Gran Sasso Science Institute, L'Aquila, Italy

CERN, Geneva Switzerland

E-mail: Carlo.Rubbia@cern.ch

XVI International Workshop on Neutrino Telescopes

2-6 March 2015

Palazzo Franchetti, Istituto Veneto, Venice, Italy

${ }^{1}$ Speaker 


\title{
Muon cooling: \\ a Higgs Factory at CERN ?
}

\author{
Carlo Rubbia \\ Gran Sasso Science Institute, L'Aquila, Italy \\ IASS, Institute for Advanced Sustainability Studies \\ Potsdam, Germany \\ Senator for life of the Italian Republic
}

\section{The LHC observation of the Higgs at $125 \mathrm{GeV}$}

- CMS and Atlas have observed a narrow line of high significance at about $125 \mathrm{GeV}$ mass. compatible with the Standard Model Higgs boson.

$>$ ATLAS: $m_{H}=125.5 \pm 0.2$ (stat) \pm 0.6 (sys) GeV

>CMS: $\quad m_{H}=125.8 \pm 0.4$ (stat) \pm 0.4 (sys) GeV

- Their data are consistent with fermionic and bosonic coupling expected from a SM Higgs particle.

- Searches have been performed in several decay modes, however in the presence of very substantial backgrounds.

- Experimental energy resolutions have been so far much wider of any conceivable intrinsic Higgs width.

- Results of both experiments also exclude other SM Higgs bosons up to approximately $600 \mathrm{GeV}$. 


\section{The discovery}

Signal and background in the $H \rightarrow 2 \gamma$ channel

CMS

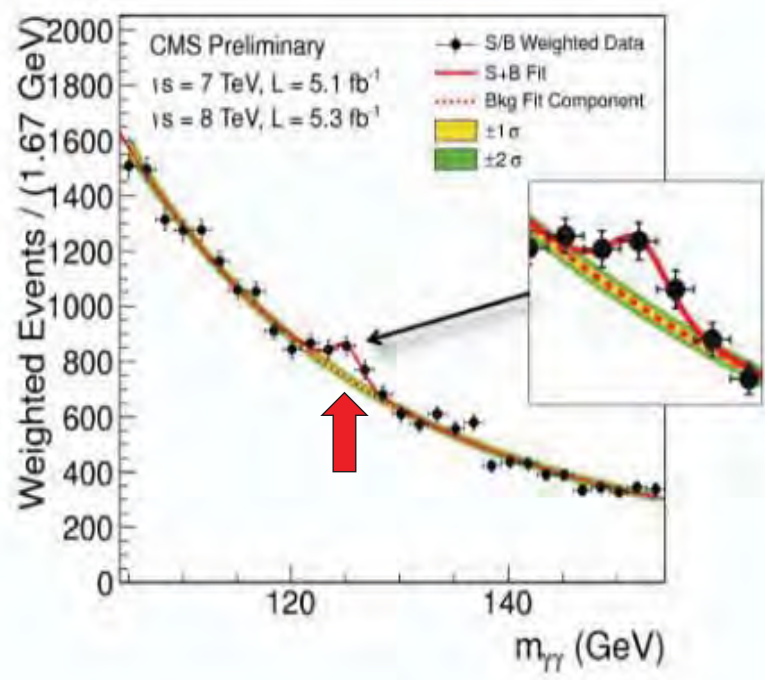

ATLAS

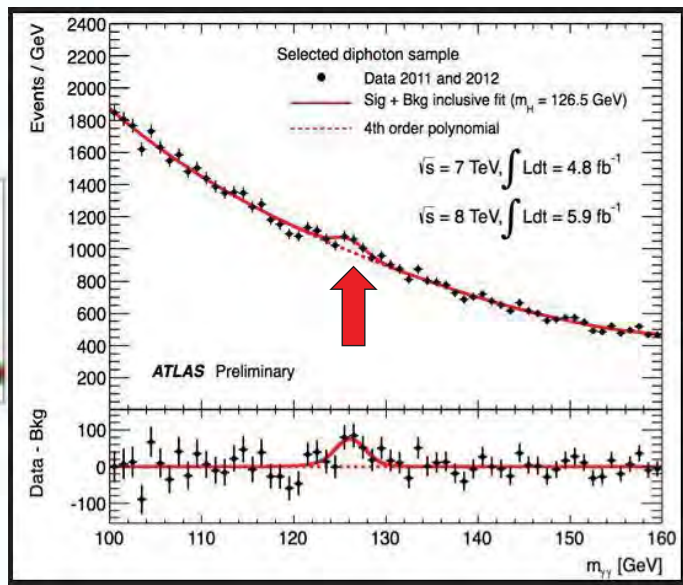

Slide\# : 3

\section{Present results}

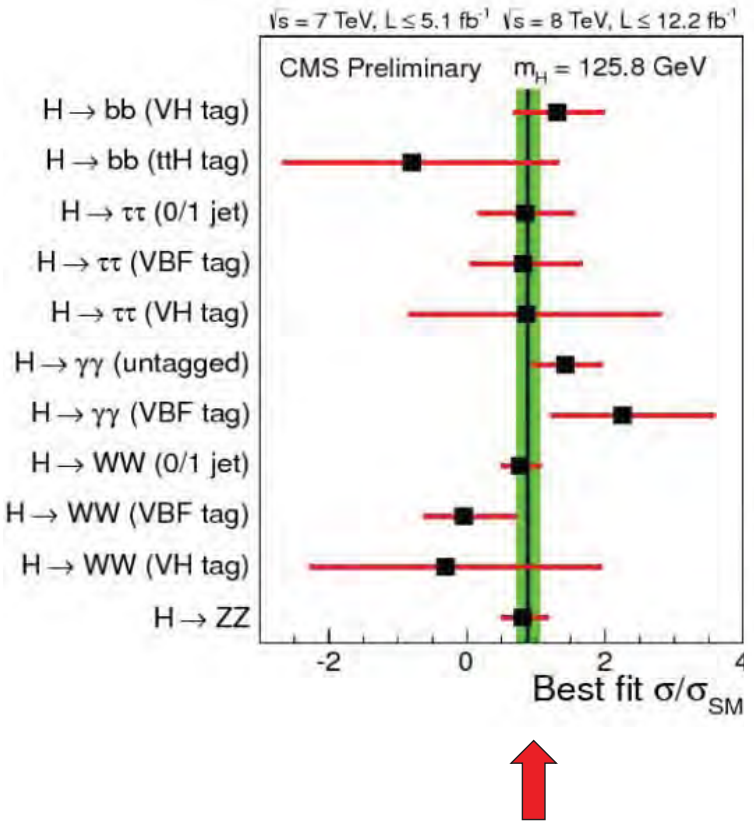

Venice,March.2015

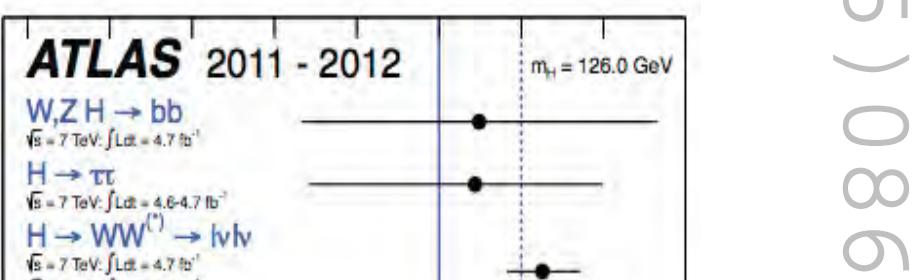

$=8$ TeV: $\int \operatorname{Lt}=5.8$ tot

$\mathrm{H} \rightarrow \gamma \gamma$

$\sqrt{8}=7$ tov $\int$ Lat $=4,8$ is

$\sqrt{8}=8$ TeV $\int \log =5,900^{\circ}$

$\mathrm{H} \rightarrow \mathrm{ZZ}^{(\prime)} \rightarrow 4$ I

$\sqrt{5}=7$ ToV: $\int \operatorname{tat}=4.8$ th

$\sqrt{3}+B$ TeV: $\int L A t-5 . B D$

Combined

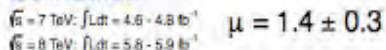

$1+1$

Signal strength $(\mu)$ 


\section{Present situation}

- For these values, the electroweak vacuum is claimed metastable, but with a lifetime longer than the age of the Universe.

- The Standard Model can be valid without new physics all the way up to the Planck scale. Thus, there may be only one standard model (SM) Higgs and no need for the "no fail theorem".

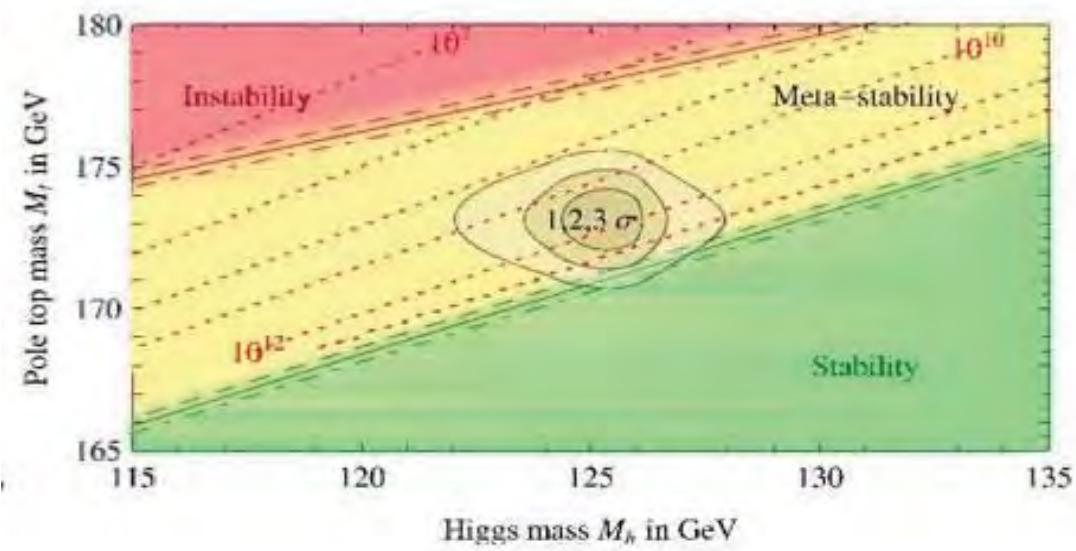

\section{The Higgs width according to the Standard Model}

- Like in the case of the Zo, the determination of the $\mathrm{H}_{0}$ width will be crucial in the determination of the nature of the particle and the underlying theory

- Cross section is shown here, convoluted with a Gaussian beam distribution.

- Signal is not affected only if the rms beam energy width is $\leq$ a few MeV.

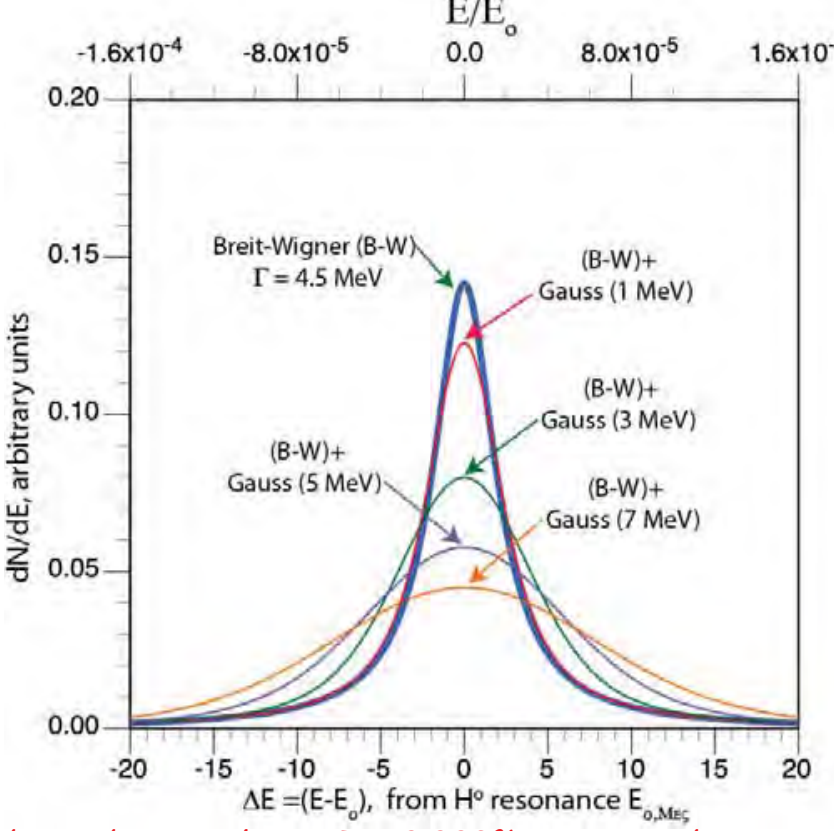




\section{Ultimate dominance due to systematic effects}

- The estimates reflect $1 \mathrm{LHC}$ detector accumulating $300 \mathrm{fb}-1$ of data, dominated at this level by systematic errors of the ATLAS and CMS collaborations and their best understanding.

- ATLAS and CMS have estimated errors also for $3000 \mathrm{fb}-1$ from the High-L LHC.

- However such estimates can hardly be a straightforward extrapolation of the current performances.

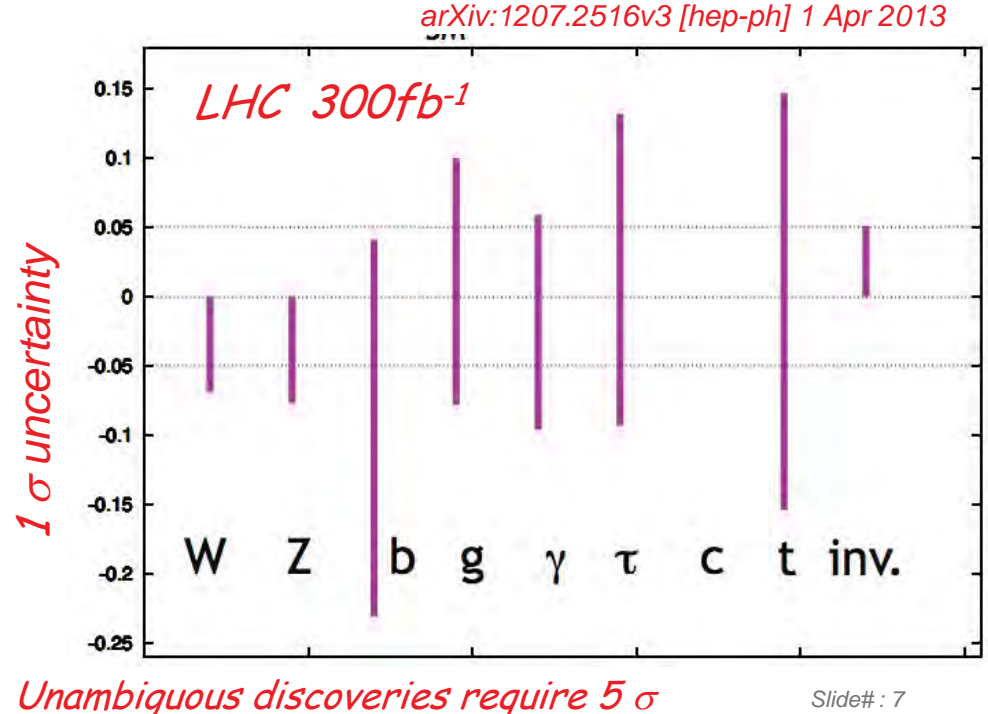

\section{The need of a better precision}

- What precision is needed in order to search for possible additional deviations from the $S M$, even under the assumption that there is no other additional "Higgs" state at the LHC?

- Predicted ultimate LHC accuracies for "exotic" alternatives

\begin{tabular}{lccc}
\hline \hline R.S. Gupta et al. & $\Delta h V V$ & $\Delta h \bar{t} t$ & $\Delta h b b$ \\
\hline Mixed-in Singlet & $6 \%$ & $6 \%$ & $6 \%$ \\
Composite Higgs & $8 \%$ & tens of $\%$ & tens of $\%$ \\
Minimal Supersymmetry & $<1 \%$ & $3 \%$ & $10 \%^{a}$, \\
LHC 14 TeV, $3 \mathrm{ab}^{-1}$ & $8 \%$ & $10 \%$ & $15 \%$ \\
\hline
\end{tabular}

Ultimate at $\angle H C$ $1 \mathrm{ab}=10^{-42} \mathrm{~cm}^{2}$

$\frac{g_{h b b}}{g_{h_{\mathrm{SM}} b b}}=\frac{g_{h \tau \tau}}{g_{h_{\mathrm{SM} \tau \tau}}} \simeq 1+1.7 \%\left(\frac{1 \mathrm{TeV}}{m_{A}}\right)^{2} \quad \operatorname{SUSY} \tan (\beta)>5$

$\frac{g_{h f f}}{g_{h_{\mathrm{SM} f f}}} \quad \frac{g_{h V V}}{g_{h_{\mathrm{SM} V V}}} \simeq 1-3 \%\left(\frac{1 \mathrm{TeV}}{f}\right)^{2} \quad$ Composite Higgs

$\frac{g_{h g g}}{g_{h_{\mathrm{SM} g}}} \simeq 1+2.9 \%\left(\frac{1 \mathrm{TeV}}{m_{T}}\right)^{2}, \quad \frac{g_{h \gamma \gamma}}{g_{h_{\mathrm{SM} \gamma \gamma}}} \simeq 1-0.8 \%\left(\frac{1 \mathrm{TeV}}{m_{T}}\right)^{2}$ Top partners

Sensitivity to "TeV" new physics for " 5 sigma" discoveries may need 1 per-cent to sub 1 -per-cent $\sigma$ accuracies on rates. 


\section{Predictive power of theory: the case of LEP}

After the p-pbar discovery of the $Z^{0}$, its detailed studies at LEP in very clean conditions have been an essential second phase. Higher order corrections have anticipated the masses of both the top quark and of the Higgs scalar.

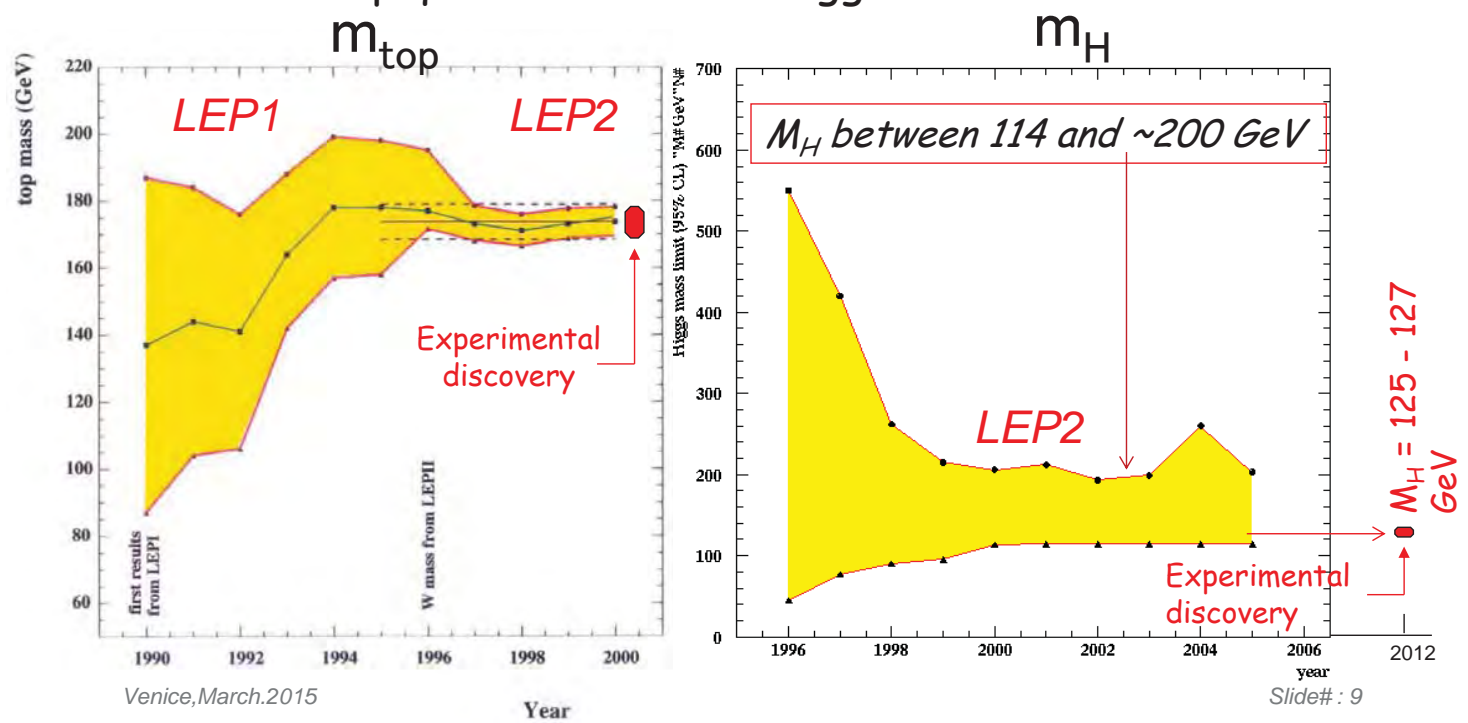

\section{Studying the Higgs beyond LHC}

- The scalar sector is definitely one of the keys to the future understanding of elementary particle physics.

- A similar second phase may be also necessary for the $H_{0}$ and the presence of structure beyond the SM may manifest itself as tiny corrections in the observation of large number of events/year in very clean experimental conditions.

Two future alternatives are hereby compared: $\quad=10^{-39} \mathrm{~cm}^{2}$

$>A e^{+} e^{-}$collider at $L>10^{34}$ and a $Z+H_{0}$ signal of $\approx 200 \mathrm{fb}$. The circumference of a new, LEP-like ring is of about $\approx 80 \mathrm{~km}$ or of a Linear Collider of $31 \mathrm{~km}$.

A $\mu^{+} \mu^{-}$collider at $L>10^{32}$ and a $H_{0}$ signal in the s-state of $\approx 20$ '000 fb. The collider radius is much smaller, only $\approx 50 \mathrm{~m}$, but the novel "muon cooling" facility is required. 


\section{Production cross sections at the e+ e-collider}

- The production cross sections of the Higgs boson with the mass of $125 \mathrm{GeV}$ for e+-e- as a function of the energy $\sqrt{s}$.

- The cross sections of the production processes as a function of the $\sqrt{s}$ collision energy.

- The Higgs-strahlung diagram (Left), the W-boson fusion process (Middle) and the topquark association (Right).

- Double Higgs boson diagrams via off-shell Higgs-strahlung (Left) and W-boson fusion (Right) processes
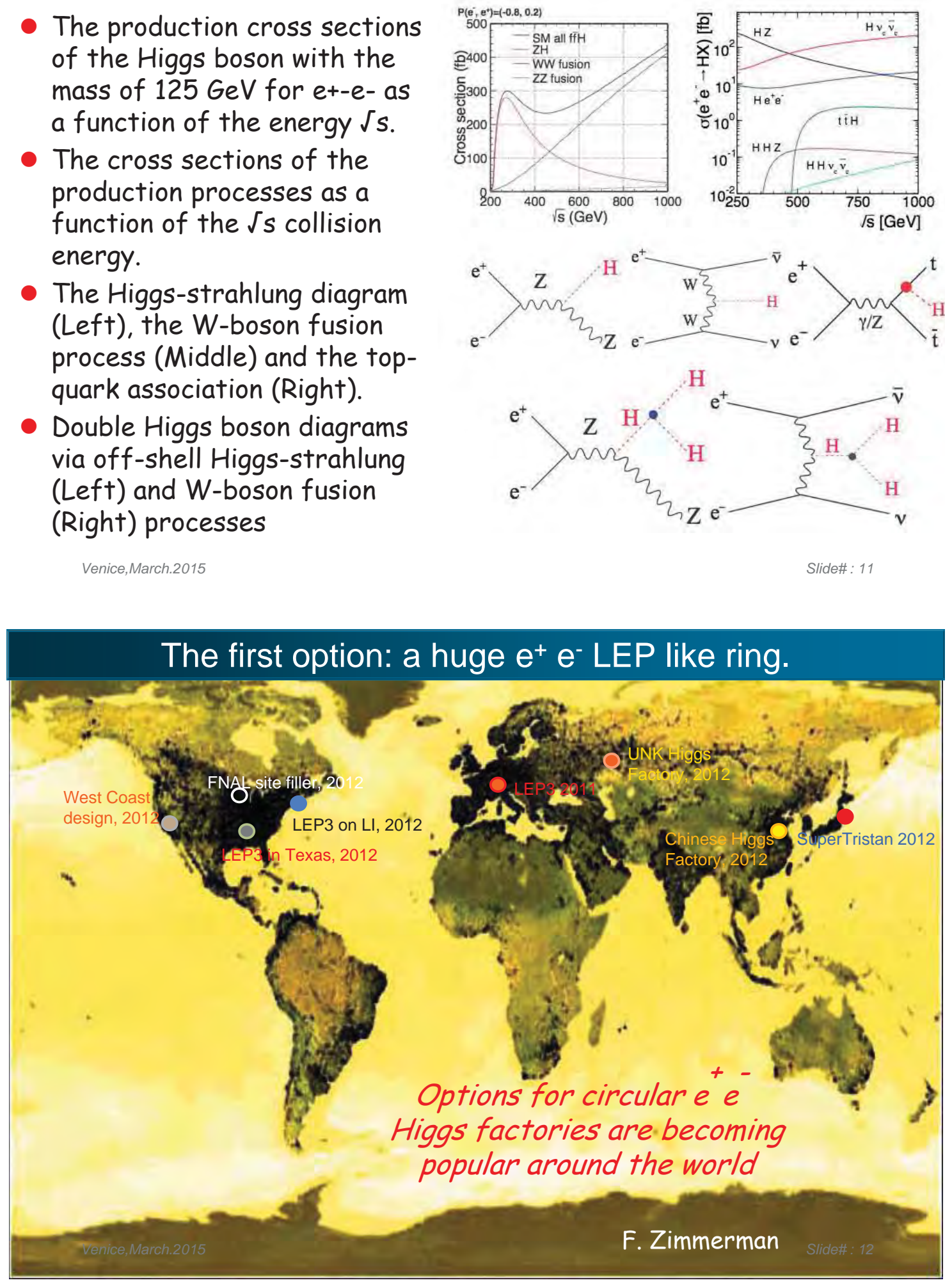


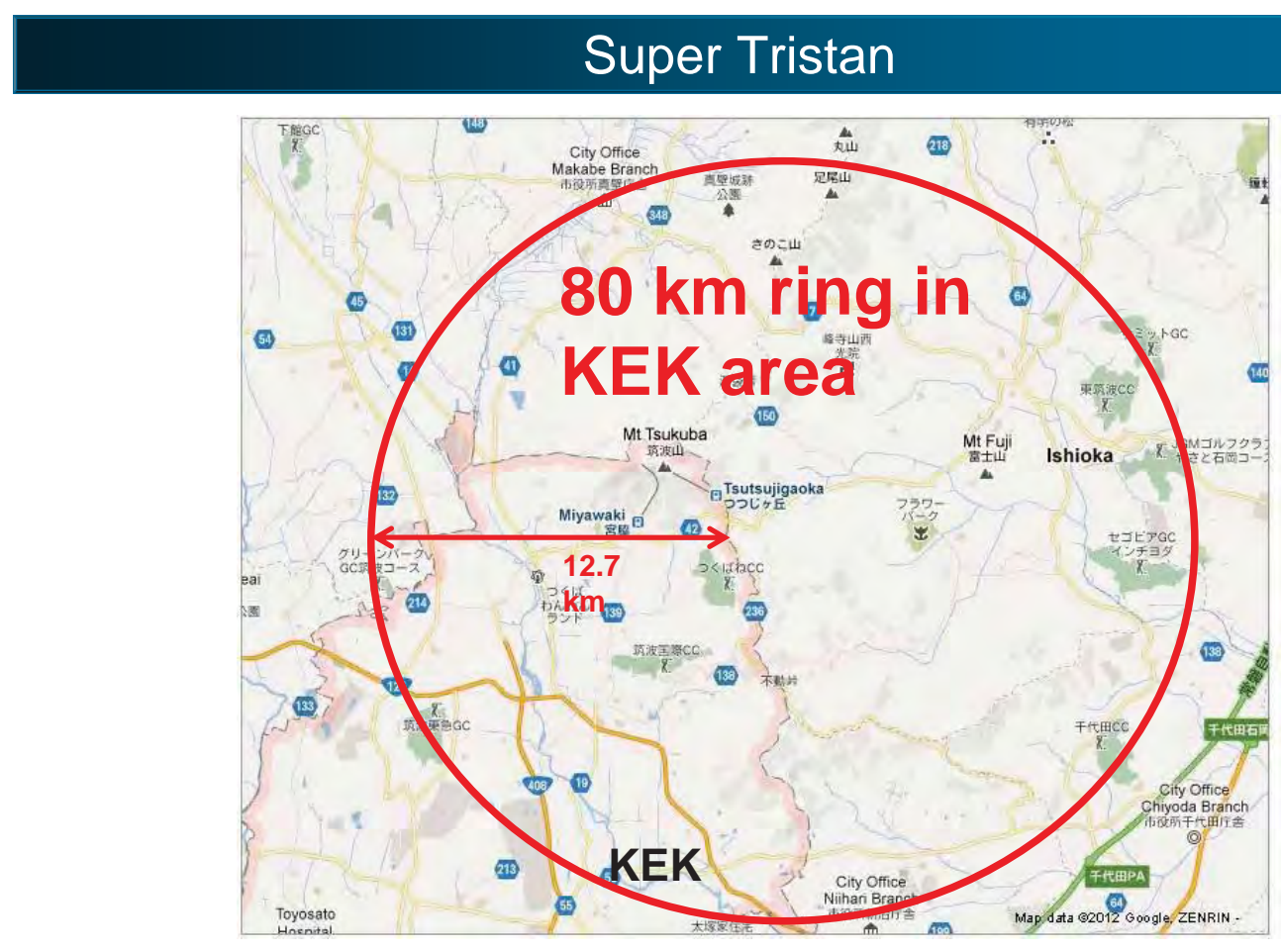

\section{TLEP tunnel in the Geneva area}

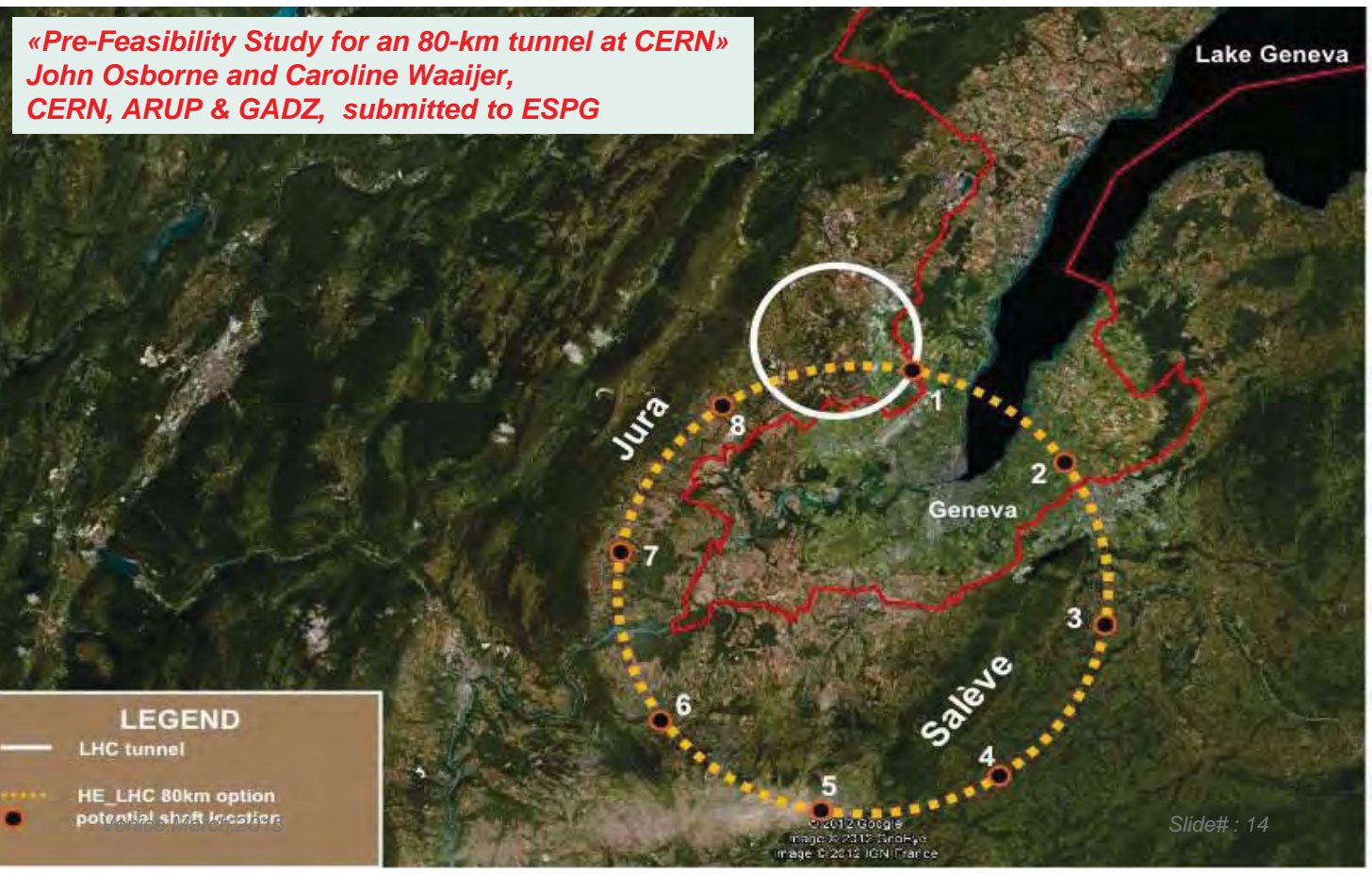




\section{Requirements for the Higgs with a $e^{+} e^{-}$collider}

- The luminosity is pushed to the beam-strahlung limit.

- Collisions are at an angle, but with fewer bunches than for a B-Factory: a nano-beam scheme

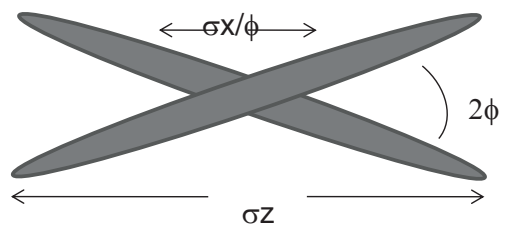

- Luminosity (several $\times 10^{34} \mathrm{~cm}^{-2} \mathrm{~s}^{-1}$ ), costs and power consumption ( $\approx 100 \mathrm{MW}$ ) are comparable to those of a linear collider ILC.

- In order to reach luminosity (factor $\approx 500 \times$ LEP2) and power consumptions ( factor $5 \times$ LEP2) the main cures are

$>$ Huge ring (80 km for SuperTristan or for T-LEP)

- Extremely small vertical emittance, with a beam crossing size the order of $0.01 \mu$ (it has been $3 \mu$ for LEP2)

- The performance is at the border of feasibility ( $\left.E_{c m} \approx 250 \mathrm{GeV}\right)$.

- However the $H_{0}$ width of $\approx 4.5 \mathrm{MeV}$ cannot be directly observed

\section{The ILC option}

- The International Linear Collider (ILC) is a high-luminosity linear electron-positron collider based on $1.3 \mathrm{GHz}$ superconducting radio-frequency (SCRF) accelerating technology.

- Its energy $\sqrt{s}$ is 200-500 GeV (extendable to 1 - TeV).

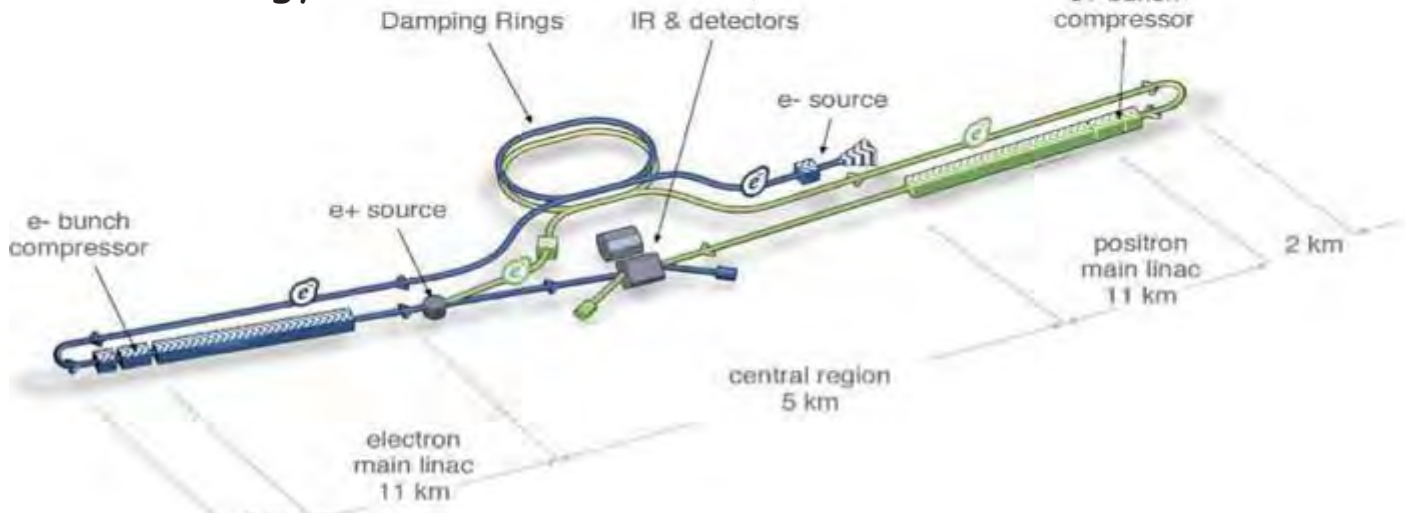

The total footprint is $\sim 31 \mathrm{~km}$.. To upgrade the machine to Ecms $=1 \mathrm{TeV}$, the linacs and the beam transport lines would be extended by another $\sim 11 \mathrm{~km}$ each. 


\section{Super-Tristan vs ILC}

Ring or Linear?
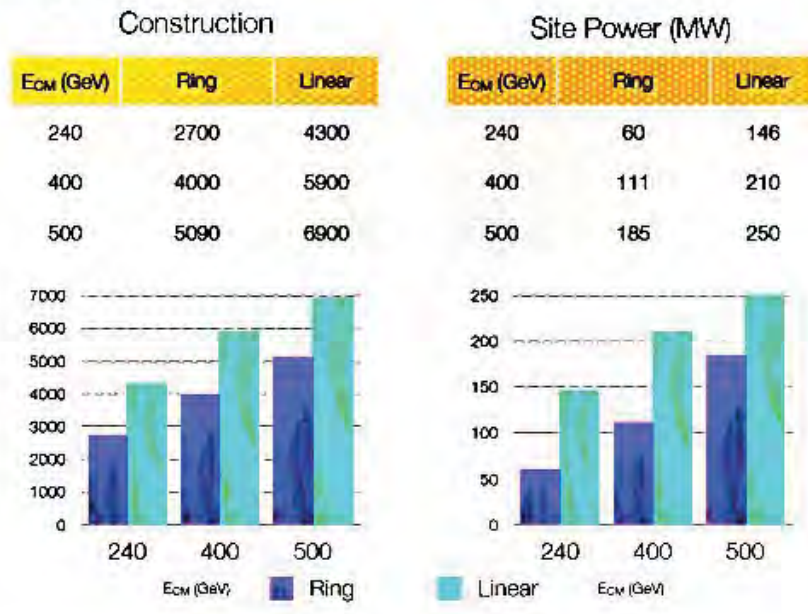

Linear collider and circular ring have comparable costs and power consumptions The more conservative ring alternative is preferred.

\section{Comparing LHC and e+-e- colliders}

Compared with th $L H C$, in order to be fully effective, the energy of an ILC should be increased progressively from $250 \mathrm{GeV}$ till 1 $\mathrm{TeV}$, with correspondingly longer structures and higher powers.

LHC $300 \mathrm{fb}-1$ $\mathrm{g}(\mathrm{hAA}) /\left.\mathrm{g}(\mathrm{hAA})\right|_{S M}-1 \mathrm{LHC} / \mathrm{ILC1} / \mathrm{ILC} / \mathrm{ILCTeV}$

Ring or ILC1 $250 \mathrm{GeV}$ $250 \mathrm{fb}-1$

ILC $500 \mathrm{GeV}$ $500 \mathrm{fb}-1$

ILC TeV 1.0 TeV $1000 \mathrm{fb}-1$

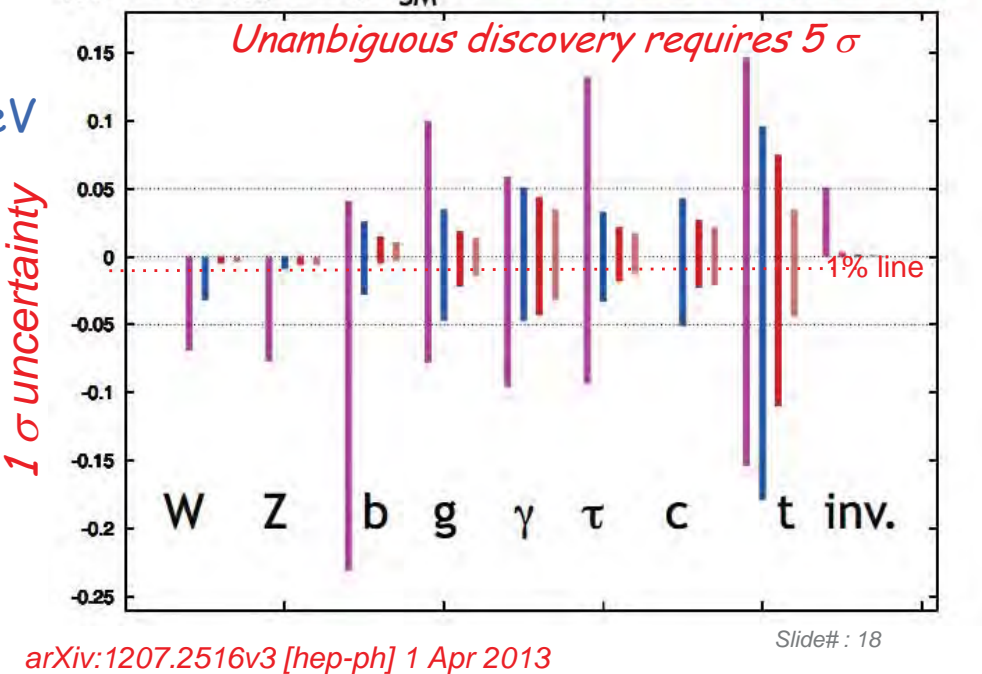




\section{The second option: a $\mu^{+} \mu^{-}$collider ?}

- The direct $H^{\circ}$ cross section is greatly enhanced in a $\mu^{+} \mu^{-}$ collider when compared to an $e^{+} e^{-}$collider, since the $s$-channel coupling to a scalar is proportional to the lepton mass.

- Like in the well known case of the $Z^{0}$ production, the $H^{\circ}$ scalar production in the s-state offers conditions of unique cleanliness

- An unique feature of such process - if of an appropriate luminosity - is that its actual mass, its very narrow width and most decay channels may be directly measured with accuracy.

- Therefore the properties of the Higgs boson can be detailed over a larger fraction of model parameter space than at any other proposed accelerator method.

- A particularly important conclusion is that it will have greater potentials for distinguishing between a standard SM and the $S M$-like $H_{0}$ of SUSY or of other than any other collider.

\section{A muon collider after the discovery of the Higgs}
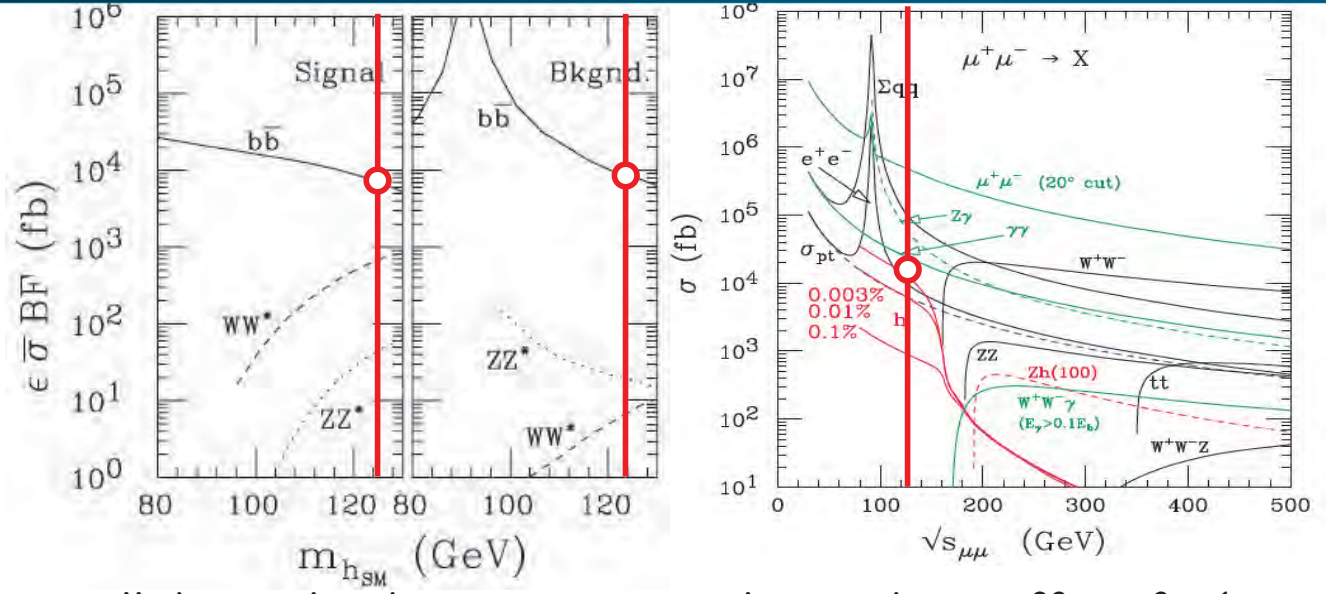

- $A \mu^{ \pm}$collider with adequate muon cooling and $L>10^{32} \mathrm{~cm}^{-2} \mathrm{~s}^{-1}$.

- Decay electron backgrounds are important: : $2 \times 10^{12} \mu^{ \pm}$decays produce $6.5 \times 10^{6}$ collimated $e^{ \pm}$decays/meter with $E_{\text {ave }} \approx 20 \mathrm{GeV}$.

- The very narrow resonant signal (4.12 MeV, $\Gamma / M_{H}=3.6 \times 10^{-5}$ for the $S M$ ) will dominate over most non resonant backgrounds. 


\section{Leading Higgs processes}

Signal and background for $\mathrm{H} \rightarrow b b, W W *$ at a energy resolution $R=0.003 \%$. folded with a Gaussian energy spread $\Delta=3.75 \mathrm{MeV}$ and $0.05 \mathrm{fb}^{-1} /$ step and with detection efficiencies included.

- Effective $\mathrm{pb}$ at the $\sqrt{s}$ resonance for two resolutions $R$ and with the $S M$ branching fractions $=\mathrm{H} \rightarrow$ bb $56 \%$ and $W W *=23 \%$

\begin{tabular}{|c|c|c|c|c|c|}
\hline \multirow{2}{*}{ R (\%) } & \multirow{2}{*}{$\begin{array}{c}\mu^{+} \mu^{-} \rightarrow h \\
\sigma_{\text {eff }}(\mathrm{pb})\end{array}$} & \multicolumn{2}{|c|}{$h \rightarrow b \bar{b}$} & \multicolumn{2}{|c|}{$h \rightarrow W W^{*}$} \\
\hline & & $\sigma_{\text {Sig }}$ & $\sigma_{B k g}$ & $\sigma_{\text {Sig }}$ & $\sigma_{B k g}$ \\
\hline 0.01 & 16 & 7.6 & \multirow{2}{*}{15} & 3.7 & \multirow{2}{*}{0.051} \\
\hline 0.003 & 38 & 18 & & 5.5 & \\
\hline
\end{tabular}

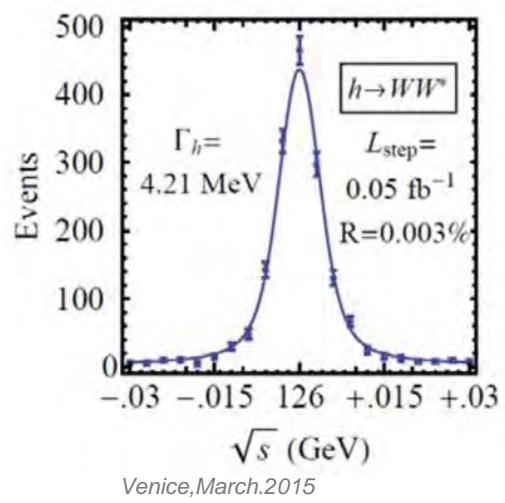

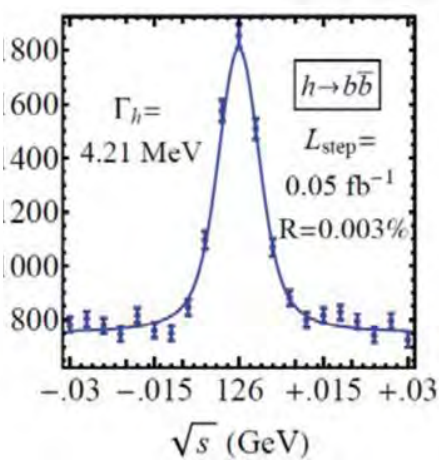

arXiv:1210.7803 [hep-ph]

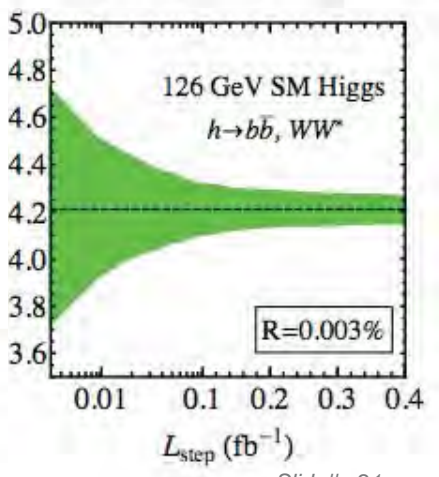

Slide\# : 21

\section{Future accelerators programs at CERN}

- A new LHC injector complex to increase the collider luminosity $10 x$ with the High Luminosity LHC (HL-LHC)).

- Two accelerators (the LP-SPL and a new $50 \mathrm{GeV}$ synchrotron, PS2) would replace the three existing ones (Linac2, the PSB, and the PS), with the injection of the SPS at $50 \mathrm{GeV}$,
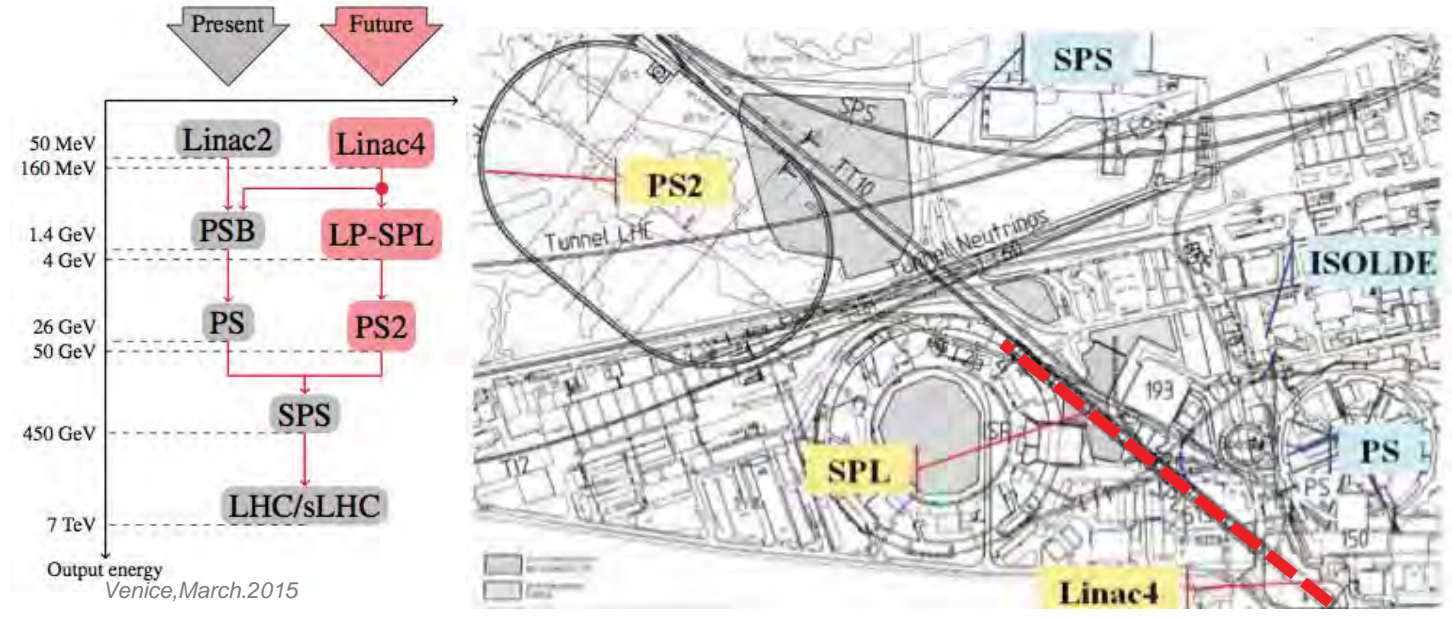


\section{CERN-SPL parameters}

- Layout of superconducting Parameter Units SPL with intermediate extractions.

SPL design is very flexible and it can be adapted to the needs of many highpower proton beam applications.

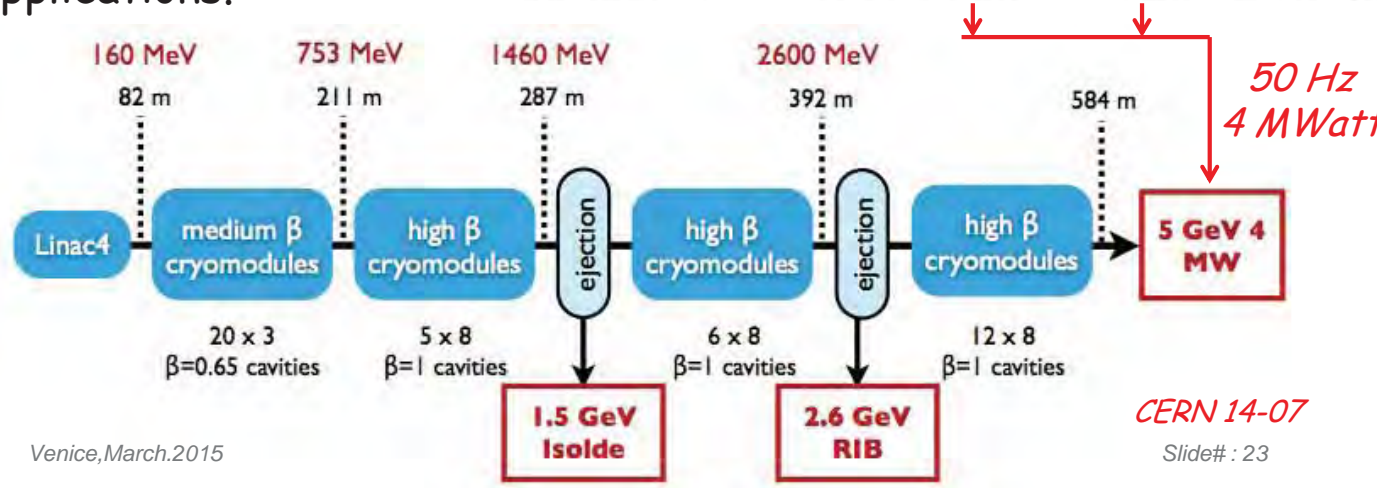

\section{A muon based Higgs factory at CERN}

A muon cooled Higgs factory can be easily housed within CERN

- The new $5 \mathrm{GeV}$ Linac will provide at $50 \mathrm{c} / \mathrm{s}$ a multi $\mathrm{MWatt}^{-}$ beam with enough pions/muons to supply the muon factory.

- The basic additional accelerator structure will be the following:

$>$ Two additional small storage rings with $\mathrm{R} \approx 50 \mathrm{~m}$ will strip $\mathrm{H}^{-}$ to a tight $p$ bunch and compress the LP-SPL beam to a few ns.

$>$ Muons of both signs are focused in a axially symmetric $B=20$ $T$ field, reducing progressively pt with a horn and $B=2 T$

$>A$ buncher and a rotator compresses muons to $\approx 250 \mathrm{MeV} / \mathrm{C}$

$>$ Muon Cooling in 3D compresses emittances by a factor $10^{6}$.

$>$ Bunches of about $2 \times 10^{12} \mu \pm$ are accelerated to $62.5 \mathrm{GeV}$

$>$ Muons are colliding in a SC storage ring of $R \approx 60 \mathrm{~m}$ (about one half of the CERN-PS, 1/100 of LHC) where about $10^{4}$ Higgs events/y are recorded for each of the experiments. 


\section{Two coupled rings to build a tight proton bunch}

- A tight $p$ bunch may be realized with a pair of rings with $\mathrm{R} \approx 50 \mathrm{~m}$ (Accumulator and Compressor).

- The $H^{-}$beam

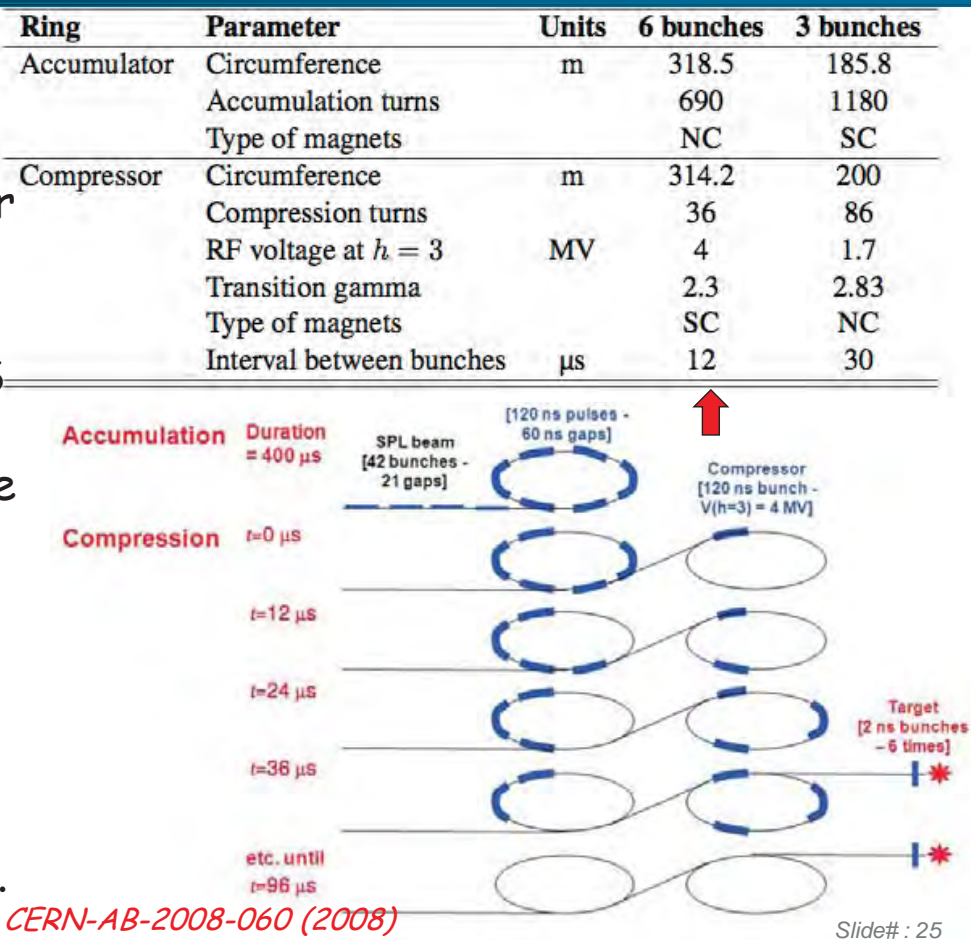

\section{Target and focussing in a axially symmetric B field}

- Liquid metal target is immersed in high field solenoid (20 T)

$>$ Proton beam is oriented with about $20^{\circ}$ with respect to axis

$>$ Particles with $p_{\dagger}<0.25 \mathrm{GeV} / \mathrm{c}$ are trapped (about $\frac{1}{2}$ of all)

$>$ Pions decay into muons

$>$ Focussing both signs of particles

- The MERIT/CERN experiment has successfully injected a Hgjet into a 15-T solenoid Pions/muons drifting as a function of $c \tau$

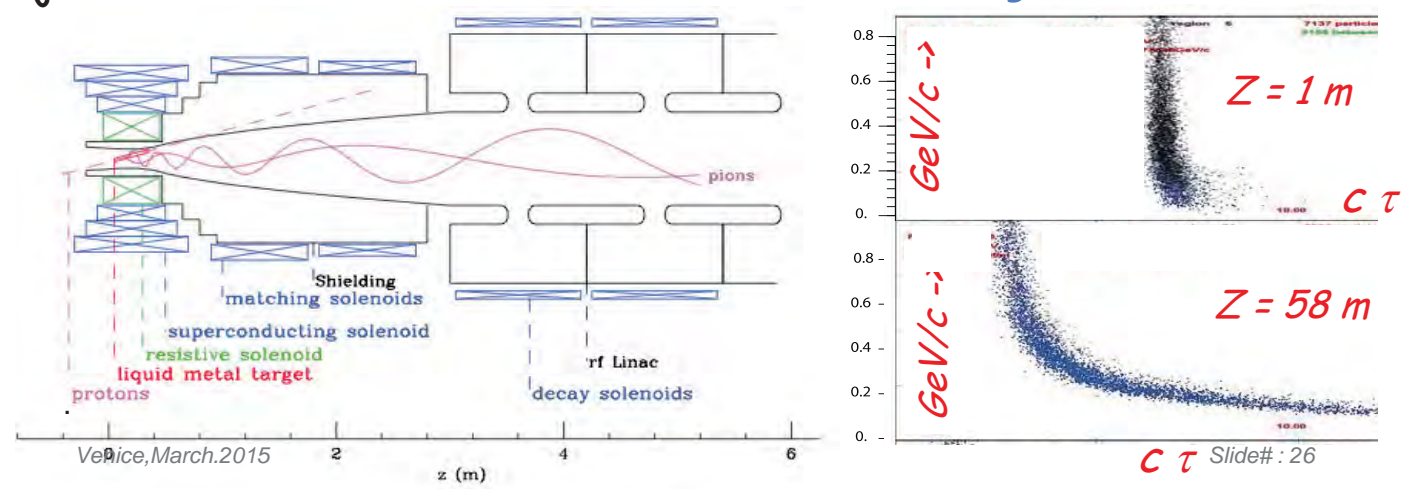




\section{Beam energy compression}

- Initially, there is a small spread in time, but a very large spread in energy. The target is followed by a drift space, where a strong correlation develops between time and energy.

- Two different methods my be used in order to provide nearly non-distorting phase rotation;:

$>260$ m of Induction linacs, see FS2 design report(BNL-52623).

$>$ Neuffer's RF bunched beams with RF rotation (IPAC 2013).

- Induction linacs reduce the r.m.s energy spread to $4.4 \%$ and after bunching to a spread to $\approx 8 \%$. In the Neuffer's scheme, the final $r$ s energy spread is $10.5 \%$.
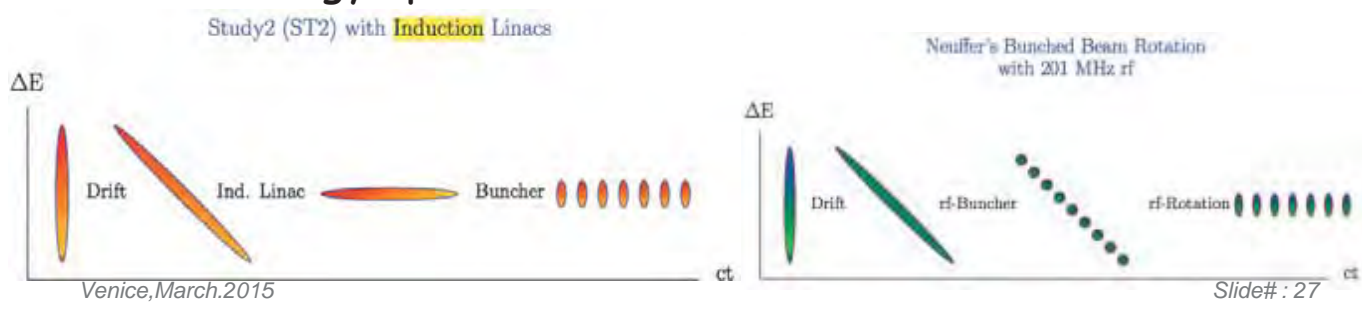

\section{Buncher and rotator to compress muon beam spectrum}

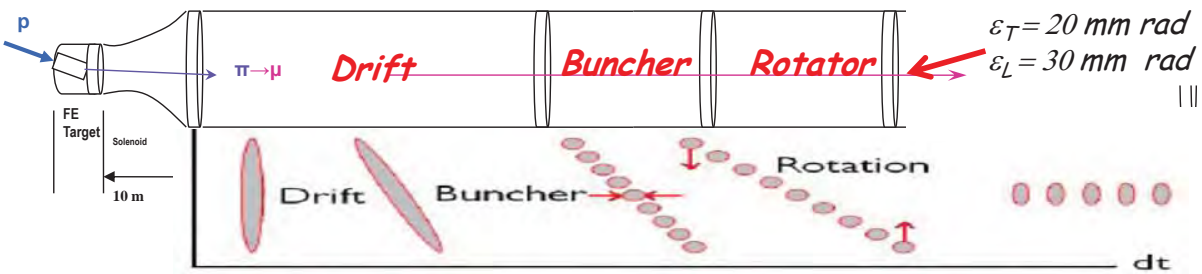

\begin{tabular}{|c|c|c|c|c|c|c}
\hline $\begin{array}{c}\text { Fromt End } \\
\text { section }\end{array}$ & $\begin{array}{c}\text { Length } \\
\boldsymbol{m}\end{array}$ & $\begin{array}{c}\# f f \\
\text { cavities }\end{array}$ & $\begin{array}{c}\text { Frequencies } \\
\text { MHz }\end{array}$ & $\begin{array}{c}\# \text { of } \\
\text { freq. }\end{array}$ & $\begin{array}{c}\text { RF } \\
\text { gradient }\end{array}$ & $\begin{array}{c}\text { RF peak power } \\
\text { requirements }\end{array}$ \\
\hline Buncher & 33 & 37 & 319.6 to 233.6 & 13 & $\begin{array}{c}4 \text { to } 8 \\
\mathrm{MV} / \mathrm{m}\end{array}$ & -1 to $3.5 \mathrm{MW} / \mathrm{frcq}$ \\
\hline Rotator & 42 & 56 & 230.210202 .3 & 15 & $\begin{array}{c}12.5 \\
\mathrm{MV} / \mathrm{m}\end{array}$ & $-2.5 \mathrm{MW} / \mathrm{cavity}$ \\
\hline
\end{tabular}

$4 \mathrm{MW}$ of protons at $5 \mathrm{GeV}$

- 50 pulses/s and $1.0 \times 10^{14} \mathrm{ppp}$

- Muons of both signs are collected

- A very efficient capture : $1.2 \times 10^{13}$ muons/pp within the 12 best bunches.

- Train of many muon bunches, requires recombination and signs

- Solenaidal coils at about $2 \mathrm{~T}$

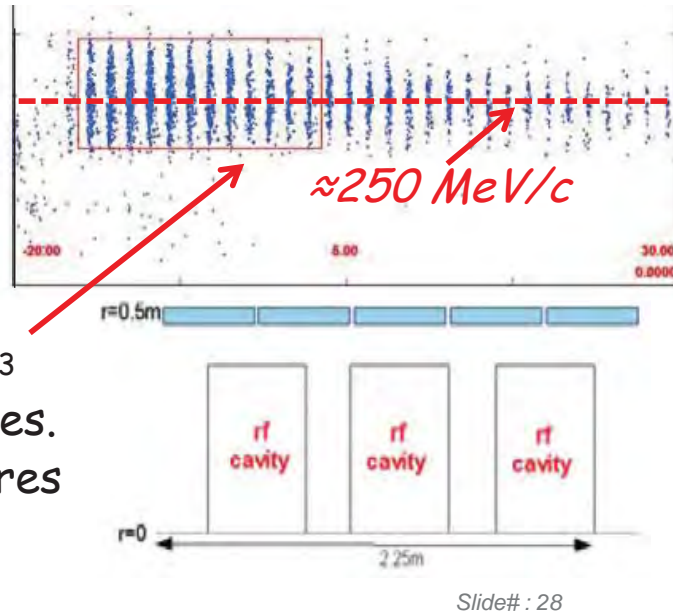




\section{Ionization cooling}

- This method, called "dE/dx cooling" closely resembles to the synchrotron compression of relativistic electrons - with the multiple energy losses in a thin, low $Z$ absorber substituting the synchrotron radiated light.

- The main feature of this method is that it produces an extremely fast cooling, compared to other traditional methods. This is a necessity for the muon case.

- Transverse betatron oscillations are "cooled" by a target "foil" typically a fraction of $\mathrm{g} / \mathrm{cm}^{2}$ thick. An accelerating cavity is continuously replacing the lost momentum.

- Unfortunately for slow muons the specific $\mathrm{dE} / \mathrm{d} x$ loss is increasing with decreasing momentum. In order to "cool" also longitudinally, chromaticity has to be introduced with a wedge shaped " $d E / d x$ foil", in order to reverse (increase) the ionisation losses for faster particles.

T. Neuffer Particle Accelerators 1983 Vol. 14 pp. 75-90

\section{Muon cooling ring: transverse emittance}

- The emittance $\varepsilon_{\mathrm{N}}$ evolves whereby $\mathrm{dE} / \mathrm{d} x$ losses are balanced by multiple scattering (Neuffer and McDonald):

$$
\frac{d \varepsilon}{d z} \approx \frac{\varepsilon}{\beta^{2} E} \frac{d E}{d z}+\frac{\beta^{*}(13.6)^{2}}{2 \beta^{3} E m_{\mu} X_{o}} \rightarrow 0 \quad \begin{gathered}
\beta^{*}=\text { beta at cross } \\
m_{\mu} \beta_{\mu}=m u \text { values }
\end{gathered} \begin{gathered}
x_{0}=\text { Rad. Length } \\
d E / d z=\text { ioniz. Loss }
\end{gathered}
$$

- The cooling process will continue until an equilibrium transverse emittance has been reached:

$$
\varepsilon_{N} \rightarrow \frac{\beta^{*}(13.6 \mathrm{MeV} / \mathrm{c})^{2}}{2 \beta_{\mu} m_{\mu}} \frac{1}{\left(X_{o} d E / d z\right)}
$$

- The equilibrium emittance $\varepsilon_{\mathrm{N}}$ and its invariant $\varepsilon_{\mathrm{N}} / \beta \gamma$ are shown as a function of the muon momentum.

- For $\mathrm{H}_{2}$ and $\beta^{*}=10 \mathrm{~cm}, \varepsilon_{\mathrm{N}} / \beta \gamma \leq 700$ $\mathrm{mm} \mathrm{mr}$ from 80 to $300 \mathrm{MeV} / \mathrm{c}$

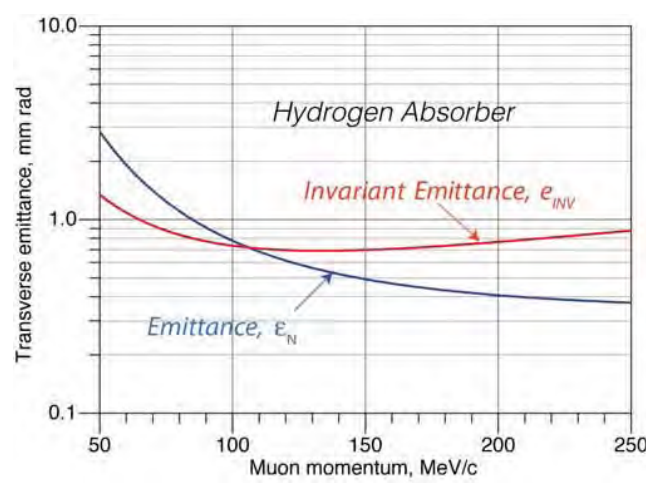




\section{Muon cooling ring: longitudinal emittance}

- Longitudinal balance is due to heat producing straggling balancing $\mathrm{dE} / \mathrm{d} \times$ cooling. $A \mathrm{dE} / \mathrm{d} \times$ radial wedge is needed in order to exchange longitudinal and transverse phase-spaces.

- Balancing heating and cooling for a Gaussian distribution limit: $\frac{d(\Delta E)^{2}}{d z}=-2(\Delta E)^{2}\left[f_{A} \frac{d}{d E}\left(\frac{d E_{o}}{d s}\right)+f_{A} \frac{d E}{d s}\left(\frac{d \delta}{d x}\right) \frac{\eta}{E \delta}\right]+\frac{d(\Delta E)_{\text {straggling }}^{2}}{d z}$

$>d E / d z \quad f_{A} d E / d s$ where $f_{A}$ is the fraction of the transport length occupied by the absorber, which has an energy absorption coefficient $d E / d s$

$>\eta$ is the chromatic dispersion at the absorber and $\delta$ and $d / d x$ are the thickness and radial tilt of the absorber

$>$ the straggling $(\mathrm{H} 2)$ is given by $\frac{d(\Delta E)_{\text {straggling }}^{2}}{d z}=\frac{\pi\left(m_{e} c^{2}\right)^{2}\left(\gamma^{2}+1\right)}{4 \ln (287) \alpha X_{o}}$

\section{Longitudinal balance (cont.)}

- The thickness of the absorber must vary with the transverse position, producing the appropriate the energy dependence of energy loss, resulting in a decrease of the energy spread

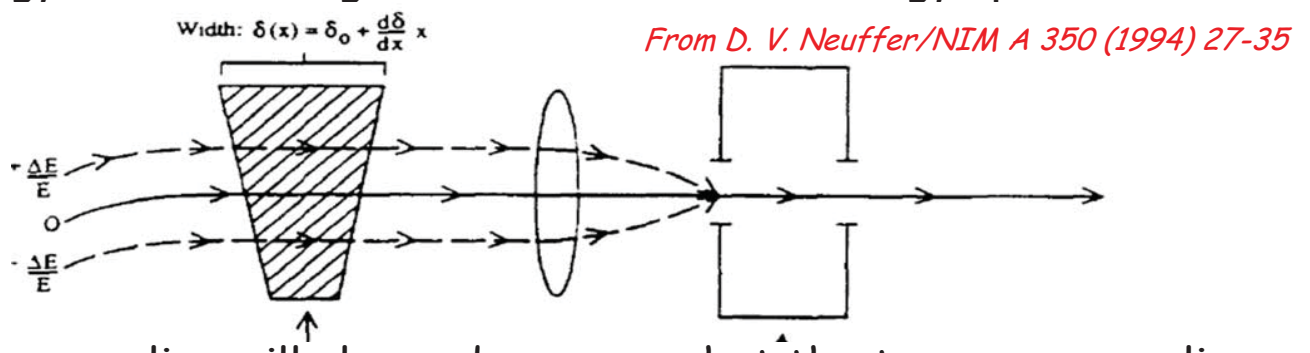

- Energy cooling will also reduce somewhat the transverse cooling, according to the Robinson's law on sum of damping decrements.

$$
2 g_{\perp}+g_{L} \cong 2
$$

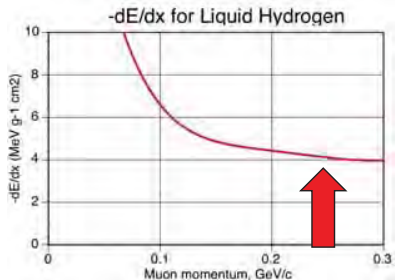

$\mathrm{dE} / \mathrm{d} x$ loss as a function of the muon momentum for hydrogen (very near to $\min$ for $250 \mathrm{MeV} / \mathrm{c}$ ) 


\section{Describing the full cooling procedure}

- Three successive steps are required in order to bring the cooling process at very low energies (initially at $\approx 250 \mathrm{MeV} / \mathrm{c}$ and later at $\approx 100 \mathrm{MeV} / \mathrm{c}$ ), after capture and bunching + rotation.

1. Linear transverse cooling of both signs and small $\Delta p$ increase.

2. Ring cooling in 6D with B brings the $\mu+$ and $\mu$ to a reasonable size Merging and cooling to single bunches

3. PIC resonance cooling. where the normal elliptical motion in $x$ $x$ 'phase space has become hyperbolic.

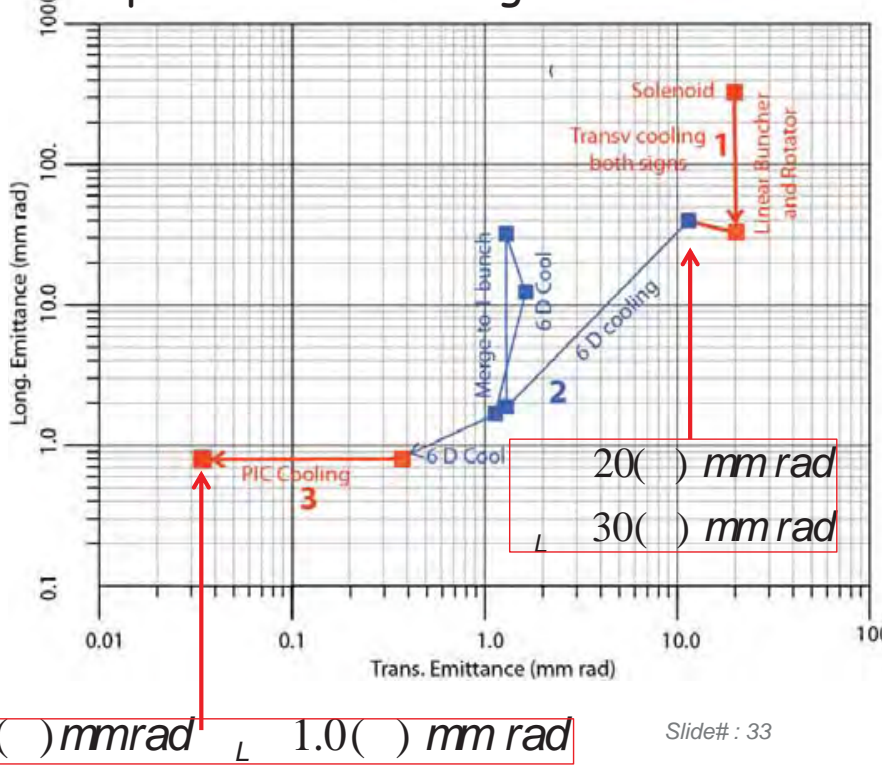

\section{1.-Linear transverse pre-cooling}

- Muons of both signs are cooled transversally with $\mathrm{LiH}$ absorbers and simultaneously accelerated with RF cavities at $200 \mathrm{MHz}$.

2 Tesla solenoidal focussing

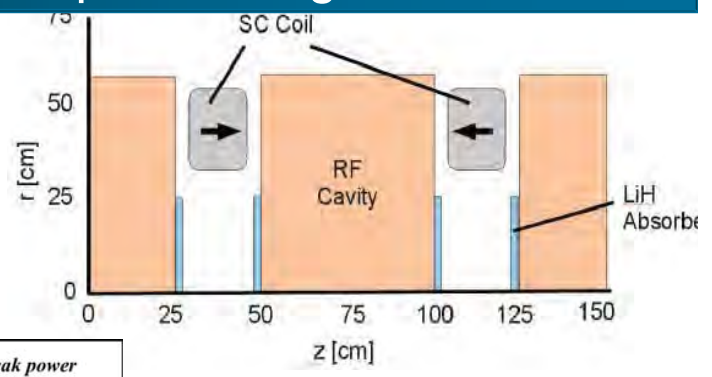

\begin{tabular}{|l|c|c|c|c|c|l|}
\hline Component & $\begin{array}{c}\text { Length } \\
\boldsymbol{m}\end{array}$ & $\begin{array}{c}\text { \#rf } \\
\text { cavities }\end{array}$ & $\begin{array}{c}\text { Frequencies } \\
\boldsymbol{M H z}\end{array}$ & $\begin{array}{c}\text { \# of } \\
\text { freq. }\end{array}$ & $\begin{array}{c}\boldsymbol{R} \text { RF } \\
\text { gradient }\end{array}$ & $\begin{array}{c}\text { RF peak power } \\
\text { requirements }\end{array}$ \\
\hline Cooler & $75 \mathrm{~m}$ & 100 & $201.25 \mathrm{MHz}$ & 1 & $16 \mathrm{MV} / \mathrm{m}$ & $\begin{array}{l}-4 \mathrm{MW} / \mathrm{cavity} \\
\text { Total peak } 400 \mathrm{MW}\end{array}$ \\
\hline
\end{tabular}

- Slight longitudinal momentum blow-up

- Method similar to the one of project MICE

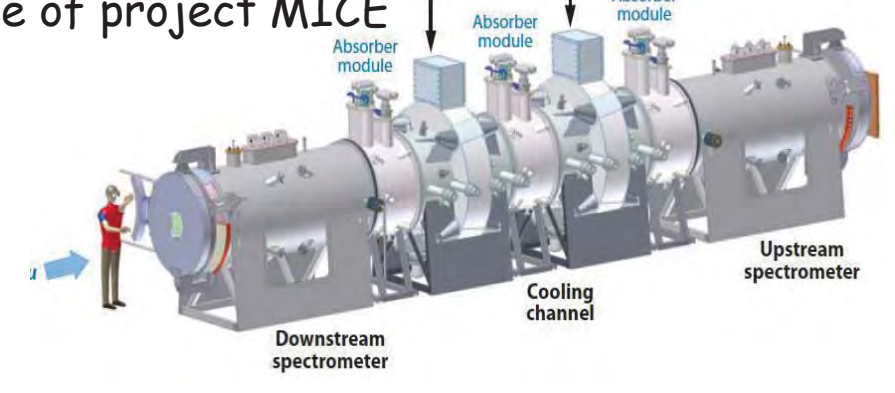




\section{2.-The cooling ring (Balbekov, Palmer)}

- An idealized muon cooling process has been numerically evaluated in 60 by Balbekov and by Palmer et al. in a small ring and for $p_{\mu} \approx 200 \mathrm{MeV}$.

In order to increase the incoming muon acceptance, strong focussing is performed with solenoids in alternate directions, rather than with q-poles (RFOFO).

\begin{tabular}{lcc}
\hline Circumference & 33 & $\mathrm{~m}$ \\
Cells & 12 & \\
Max Bz & 2.7 & $\mathrm{~T}$ \\
Coil Tilts & 2.6 & $\mathrm{deg}$. \\
Ave Momentum & 220 & $\mathrm{MeV} / \mathrm{c}$ \\
Min Trans. Beta & $35-40$ & $\mathrm{~cm}$ \\
Dispersion & 8 & \\
Wedge Material & $\mathrm{H}_{2}$ or LiH & \\
Central thickness & 28.6 & $\mathrm{~cm}$ \\
Wedge angle & 100 & $\mathrm{deg}$ \\
RF Cavities/cell & 6 & \\
Frequency & 201.25 & $\mathrm{Mhz}$ \\
Gradient & 12 & $\mathrm{MV} / \mathrm{m}$ \\
\hline March.2015 & &
\end{tabular}

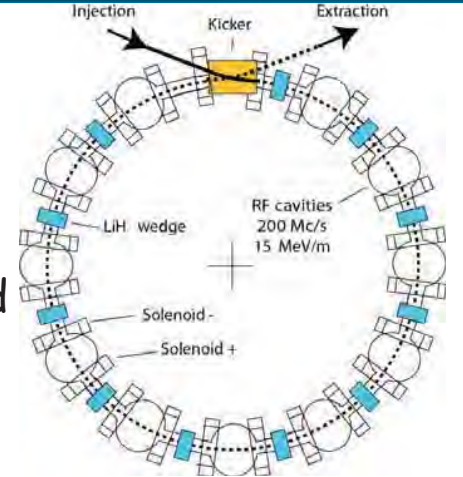

\section{Performance of Palmer et al. design}

- A first estimate of the expected cooling process is given. This is not an engineering design: for instance injection, extraction, etc. have still to be evaluated.

- The so called "merit factor" in the 6D takes into account the fractional loss of muons in the process and due to decays.
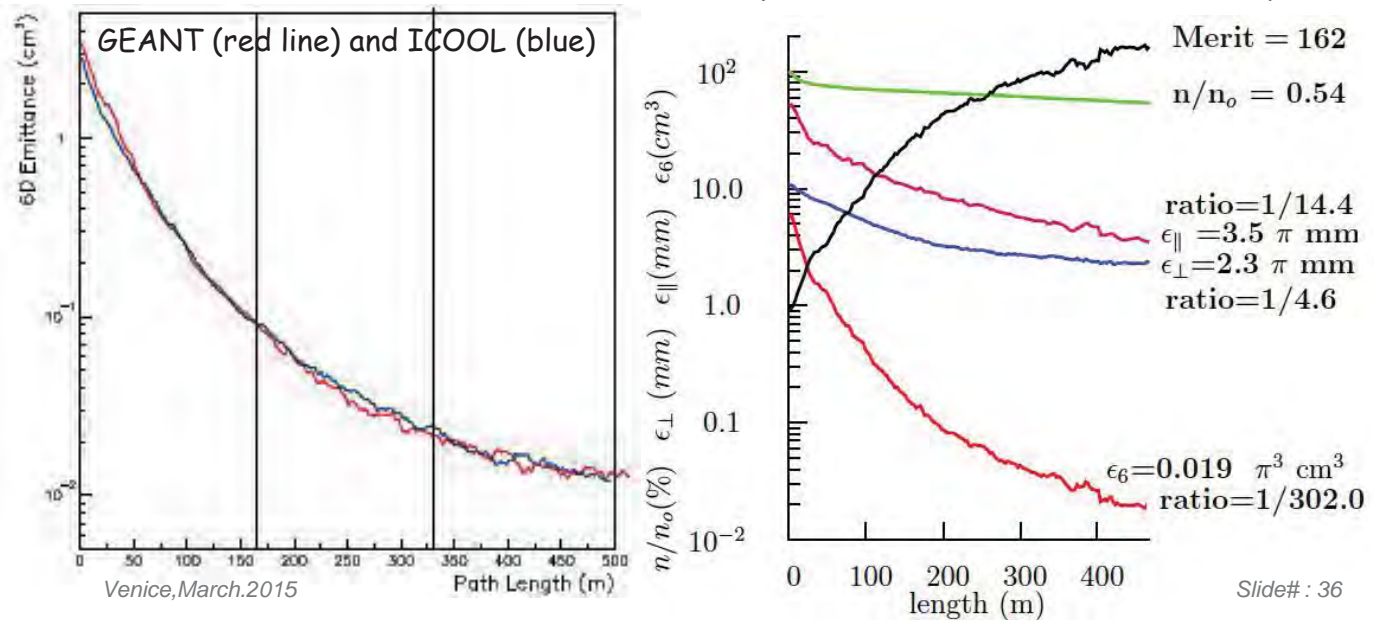


\section{3.-PIC, the Parametric Resonance Cooling of muons}

- Combining ionization cooling with parametric resonances is expected to lead to muon with much smaller transv. sizes.

- A linear magnetic transport channel has been designed by Ya.S. Derbenev et al where a half integer resonance is induced such that the normal elliptical motion of particles in $x-x^{\prime}$ phase space becomes hyperbolic, with particles moving to smaller $x$ and larger $x^{\prime}$ at the channel focal points.

- Thin absorbers placed at the focal points of the channel then cool the angular divergence by the usual ionization cooling.

LEFT ordinary oscillations RIGHT hyperbolic motion induced by perturbations near an (one half integer) resonance of the betatron frequency.

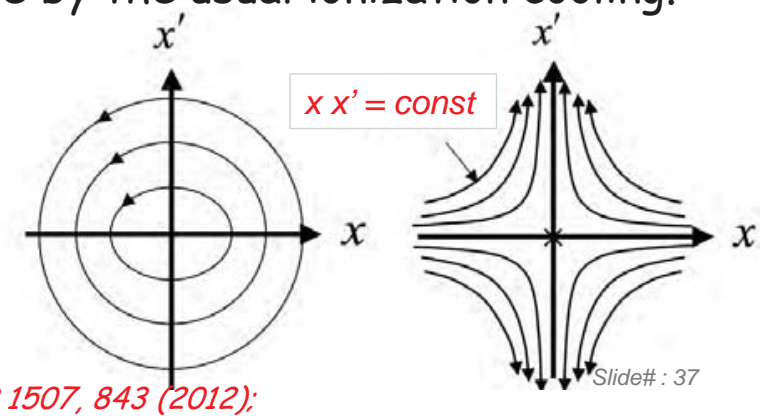

\section{Details of PIC}

- Without damping, the beam dynamics is not stable because the beam envelope grows with every period. Energy absorbers at the focal points stabilizes the beam through the ionization cooling.

- The longitudinal emittance is maintained constant tapering the absorbers and placing them at points of appropriate dispersion, vertical $\beta$ and two horizontal $\beta$.

- Comparison of cooling factors (ratio of initial to 11 final 6D emittance) with and without the PIC condition vs number of cells: more than 10x gain
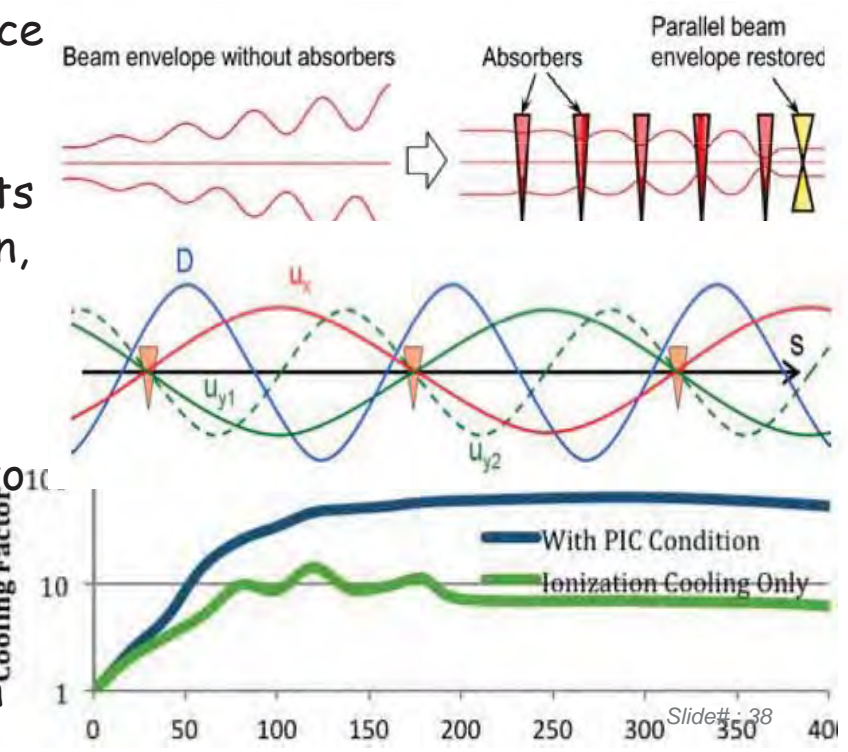


\section{Bunch acceleration to $62.5 \mathrm{GeV}$}

- In order to realize a Higgs Factory at the known energy of 126 $\mathrm{GeV}$, an acceleration system is progressively rising the energy of captured muons to $m_{H_{0}} / 2$, with the help of a series of several recirculating RLAs.

- Adiabatic longitudinal Liouvillian damping from $p \approx 0.10 \mathrm{GeV} / \mathrm{c}$ to $p_{f}=62.5 \mathrm{GeV} / \mathrm{c}$.

- Recirculating energy gain/pass, tentatively $=62.5 / 8=7.75 \mathrm{GeV}$

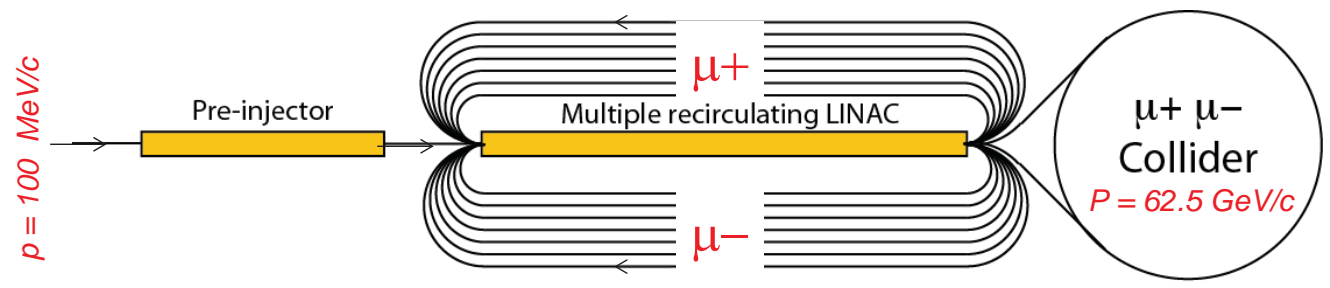

\section{6.-Muons collide in a storage ring of $\mathrm{R} \approx 60 \mathrm{~m}$}

- Lattice structure at the crossing point, including local chromaticity corrections with $\beta_{x}=\beta_{y}=\beta^{\star}=5 \mathrm{~cm}$.

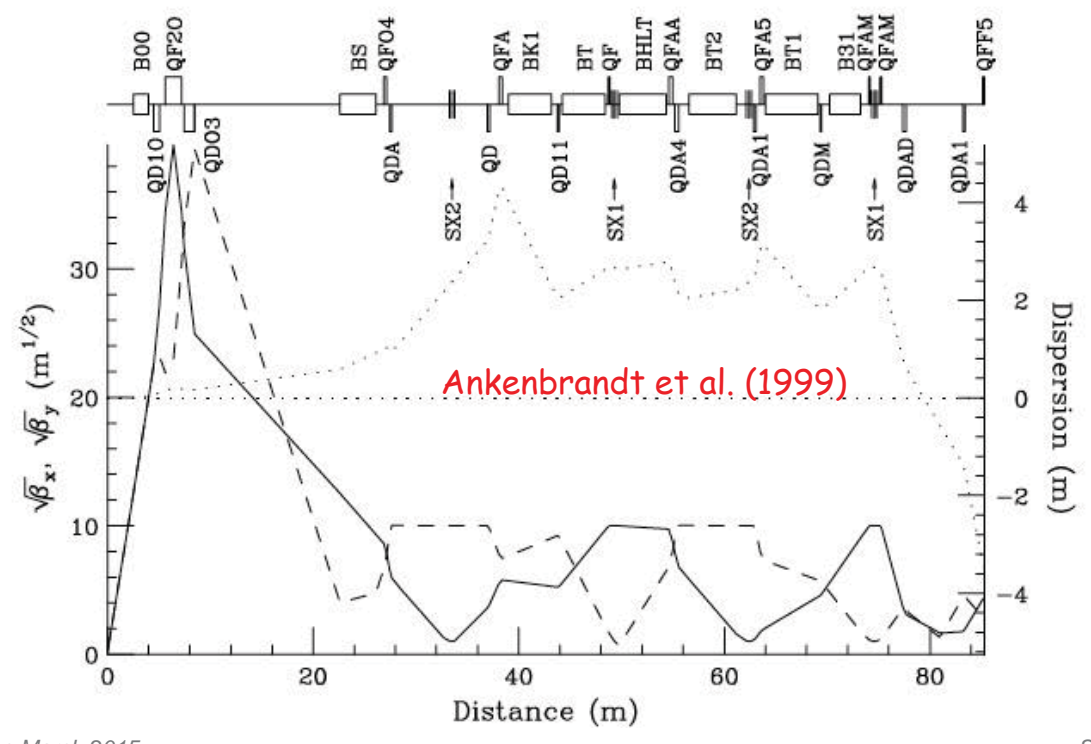




\section{Eatimated performance of the $\mathrm{H}^{\mathrm{O}}$-factory}

- Two asymptotically cooled $\mu$ bunches of opposite signs collide in two low-beta interaction points with $\beta^{\star}=5 \mathrm{~cm}$ and a free length of about $10 \mathrm{~m}$, where the two detectors are located.

- The bunch transverse rms size is $0.05 \mathrm{~mm}$ and the $\mu-\mu$ tune shift is 0.086 .

- A luminosity of $5 \times 10^{32} \mathrm{~cm}^{-2} \mathrm{~s}^{-1}$ is achieved with $1 \times 10^{12} \mu /$ bunch.

- The SM Higgs rate is $\approx 44^{\prime} 000$ ev/year in each detector.

- An arrangement with at least two detector positions is reccomanded

\begin{tabular}{|l|l|l|}
\hline Proton energy & 5 & $\mathrm{GeV}$ \\
\hline Proton power & $\mathbf{4}$ & $\mathbf{M ~ W}$ \\
\hline Event rate & 50 & $\mathrm{c} / \mathrm{s}$ \\
\hline Protons/pulse & $10^{\wedge} 14$ & $\mathrm{ppp}$ \\
\hline Muons, each sign & $6 \times 10^{\wedge} 12$ & $\mathrm{pp}$ \\
\hline Cooled fraction & 0.16 & \\
\hline Final momentum & 62.5 & $\mathrm{GeV} / \mathrm{c}$ \\
\hline Final gamma & 589.5 & \\
\hline Final muon lifetime & 1.295 & $\mathrm{~ms}$ \\
\hline Colliding, each sign & $\mathbf{1 \times 1 0 ^ { \wedge } \mathbf { 1 2 }}$ & $\mathbf{p p}$ \\
\hline Collider circumf. & 360 & $\mathrm{~m}$ \\
\hline Transverse emittances & 0.04 & $\mathrm{~mm} \mathrm{rad}$ \\
\hline Bunch transv, rms & 51. & $\mu$ \\
\hline Long emittance & 1 & $\mathrm{~mm} \mathrm{rad}$ \\
\hline No of turns & 1110 & \\
\hline No effective turns & 555 & \\
\hline Crossing/sec & 27760 & \\
\hline Luminosity & $\mathbf{5 \times 1 0 ^ { \wedge } \mathbf { 3 2 }}$ & $\mathbf{c m}-\mathbf{2 ~ s - 1}$ \\
\hline Cross section & $1.0 \times 10^{\wedge}-35$ & $\mathrm{~cm} 2$ \\
\hline Evly(10^7 s) & $\mathbf{4 4} \mathbf{0 0 0}$ & \\
\hline
\end{tabular}

\section{Finding the location of the Higgs}

- Presently the Higgs mass is known to some $600 \mathrm{MeV}$. It will be known to $\approx 100 \mathrm{MeV}$ from the LHC with $300 \mathrm{fb}-1$. But at a muon collider we need to find $M_{H}$ to $\sim 4 \mathrm{MeV}$ and then select the resonance location.

- Finding the Higgs requires a few months running at $1.7 \times 10^{31}$ luminosity.

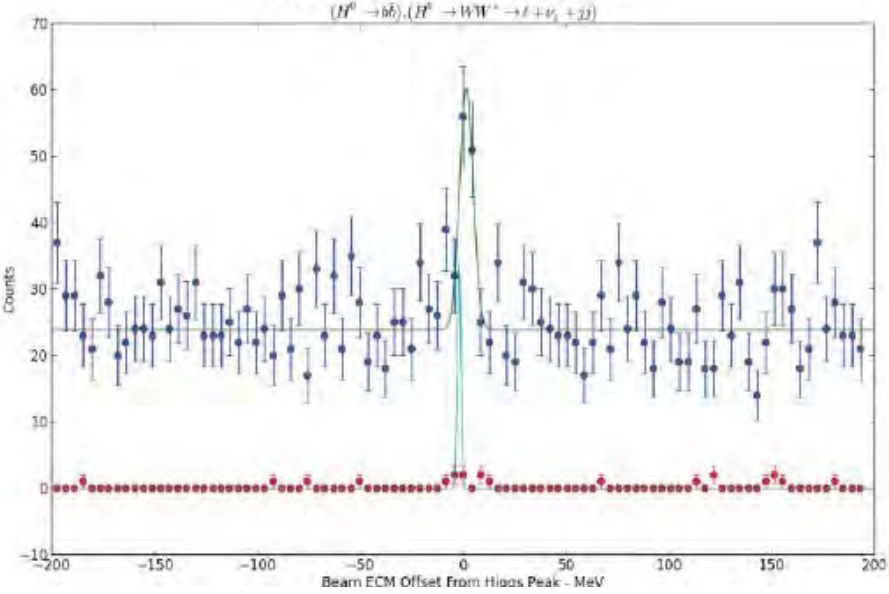




\section{Polarization \& Energy measurement}

- The effects of polarization are visible in the single electron decay angular distributions.

$$
\begin{gathered}
<E_{\text {lab }}>=\frac{7}{20} E_{\mu}\left(1+\frac{\beta}{7} \hat{P}\right) \\
E(t)=N e^{(-\alpha t)}\left(\frac{7}{20} E_{\mu}\left(1+\frac{\beta}{7}(\hat{P} \cos \omega t+\phi)\right)\right)
\end{gathered}
$$

- Measure 7 from fluctuations in electron decay energies

- The e-rate is extremely high

- Since frequencies can be very precisely measured, energy $E$, and $\delta E$ can be measured to a few hundred keV or even better

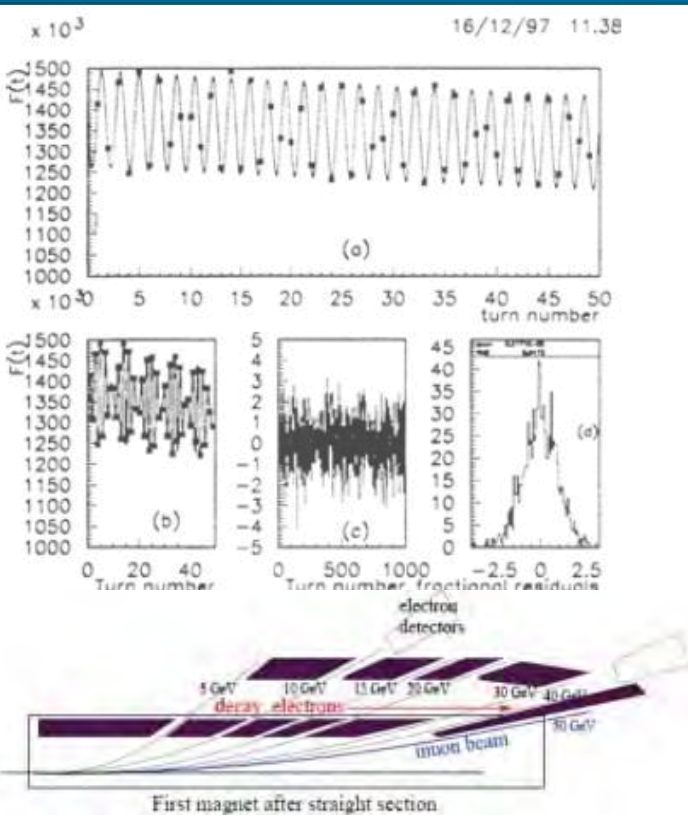

Muon spectrometer Slide\# : 43

\section{Muon related backgrounds:an open problem}

- A major problem is caused by muon decays, namely electrons from $\mu$ decay inside the detector with $\approx 2 \times 10^{3} \mathrm{e} / \mathrm{meter} / \mathrm{ns}$, however collimated within an average angle of $10^{-3} \mathrm{rad}$.

- A superb collimation is required with the help of absorbers in front of the detector's straight sections.

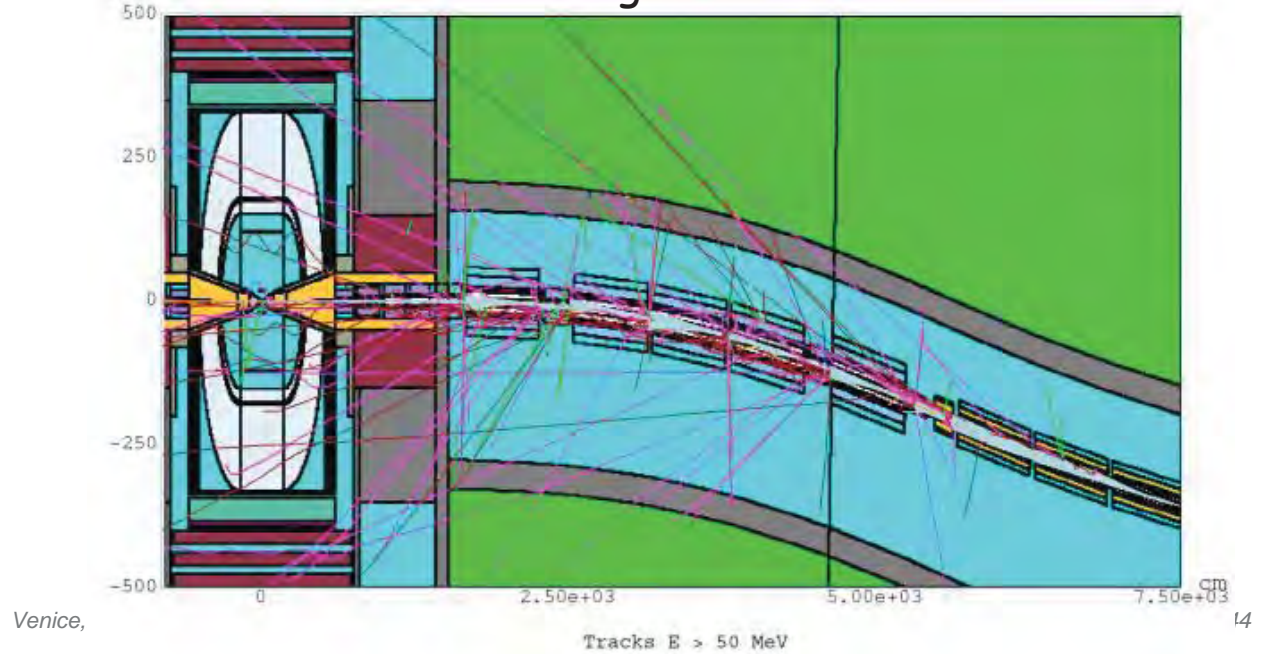


The muon Higgs collider:

\section{Advantages}

Large cross section $\sigma\left(\mu^{+} \mu \rightarrow h\right)=41 \mathrm{pb}$ in s-channel resonance, compared to $e^{+} e^{-} \rightarrow Z H$ with $0.2 \mathrm{pb}$ at $250 \mathrm{GeV}$.

> Small size footprint: it may fit in the CERN site $>$ Cost so far unknown but far smaller than the ILC.

$>$ No synchrotron radiation and beamstrahlung problems

$>$ Precise measurements of line shape and total decay width $\Gamma$ - Exquisite measurements of all channels and tests of SM.

$\rightarrow$ A low cost demonstration of muon cooling can be done first.

\section{Challanges}

Muon 2D and 3D cooling needs to be demonstrated

$>$ Need ultimately very small c.0.m energy spread (0.003\%)

$>$ Backgrounds from constant muon decay

$>$ Significant $R \& D$ required towards end-to-end design

\section{The next stejp: the refallzation or the Initual Cooling} Exneriment

- Physics requirements and the studies already undertaken with muon cooling suggest that the next step, prior to but adequate for a specific physics programme could be the practical realization of a full scale cooling demonstrator.

- Indicatively this corresponds to the realization of a cascade of unconventional but very small rings of few meters radius, in order to achieve the theoretically expected longitudinal and transverse emittances of asymptotically cooled muons.

- The injection of muons from pion decays could be extracted from some existing accelerator at low intensity.

- The goal is of experimentally demonstrate the full 6D cooling

- The other facilities, namely (1) the pion/muon production, (2) the final, high intensity cooling system (3) the subsequent muon acceleration and (4) the accumulation in a storage ring could be constructed later and only after the success of the initial cooling experiment has been confirmed. 


\section{Exemplificative initial cooling experiment}

A given sign muon cooling arrangement ( $\mu+$ or $\mu-)$ is required and with few particles in a very short pulse and two rings.

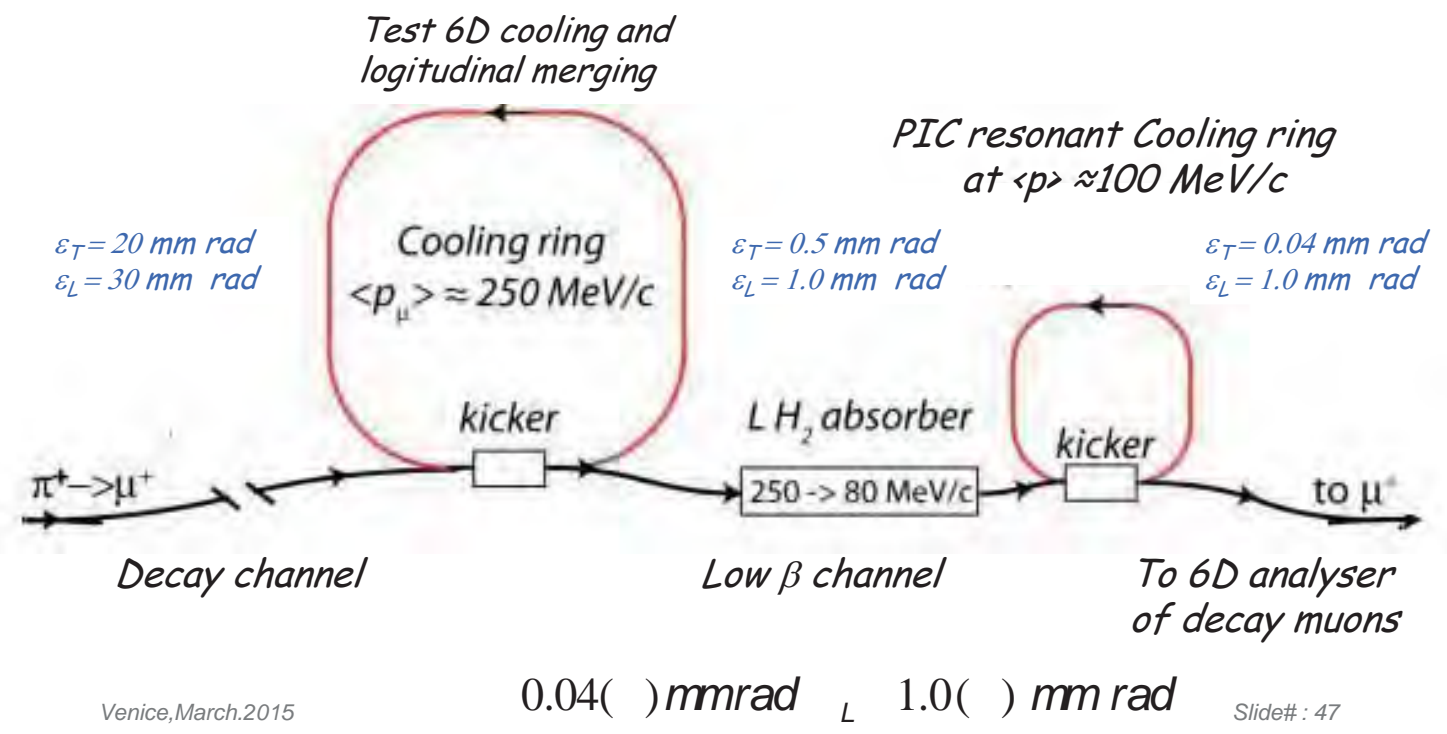

\section{A straightforward design for the achromatic cooling ring}

- A realistic study is the one of Garren et al. (NIM, 2011).

- The four-sided ring has four $90^{\circ}$ arcs with 8 dipoles separated by solenoids.

- Arcs are achromatic both horizontally and vertically. The dispersion is zero in the straight sections between the arcs.

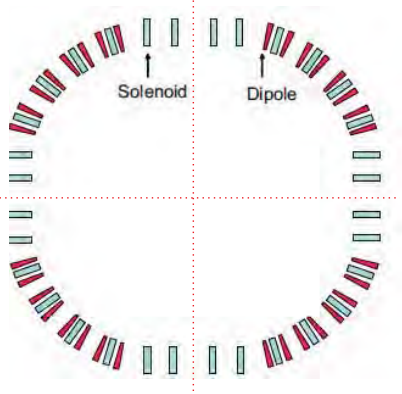

Injection/extraction kickers are used in a straight section; a superconducting flux pipe is used for the injected beam.

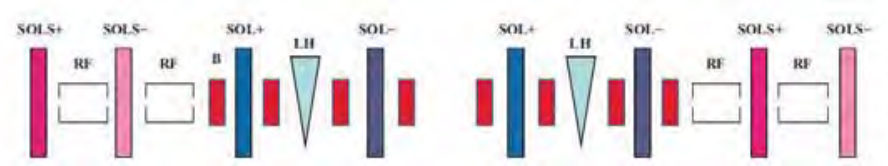

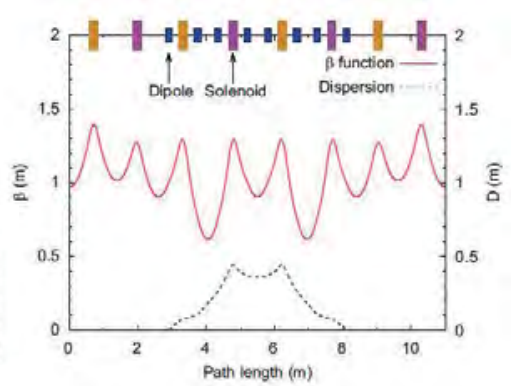




\section{The proposed initial cooling experiment}

- A first "wide band" cooling ring must collect the widest muon spectrum peaked around $250 \mathrm{MeV} / \mathrm{C}$ and to introduce a first major reduction in the transverse and longitudinal emittances, namely:

$>$ solenoids instead of quadrupoles have a wider acceptance

> with a few turns, only integer resonances are harmful

$>$ As a first cooler, the ionization absorber does not have to be made with $\mathrm{LH}_{2}$ : other solid materials $(\mathrm{LiH})$ may be used.

- An intermediate $\mathrm{LH}_{2}$ absorber $\approx 3 \mathrm{~m}$ long inside a low $\beta *$ channel reduces the vector muon momenta by range.

- The resulting beam must then be extracted and its momentum substantially reduced to about $100 \mathrm{MeV} / \mathrm{C}$.

- A second "deep freezer" cooling PIC ring must ensure an required asymptotic beam emittances

- The first muon cooling ring should present no unexpected behaviour and good agreement between calculations and experiment is expected both transversely and longitudinally

- The novel Parametric Resonance Cooling (PIC) involves instead the balance between a strong resonance growth and ionization cooling and it may involve significant and unexpected conditions which are hard to predict.

- Therefore the experimental demonstration of the cooling must be concentrated on such behaviour.

- On the other hand the success of the novel Parametric Resonance Cooling is a necessary premise for a viable luminosity of the initial proton parameters of the future CERN accelerators since the expected Higgs luminosity is proportional to the inverse of the transverse emittances, hence with about one order of magnitude of increment expected from PIC. 


\section{Conclusions}

- The recent discovery of the Higgs particle of $125 \mathrm{GeV}$ at CERN has highlighted the unique features of the direct production of a $\mathrm{H}^{\circ}$ scalar in the s-state, in analogy with the two steps of the $Z$ with the PbarP and LEP programmes and where the mass, total and partial widths of the $H^{\circ}$ can be directly measured with a remarkable accuracy and a very large number of events.

- A high energy $\mu^{+} \mu^{-}$-collider is the only possible circular high energy lepton Higgs collider that can be easily situated within the existing CERN (or FNAL) sites.

- A first step to could be the practical and experimental realization of a full scale cooling demonstrator, a relatively modest and low cost system but capable to conclusively demonstrate "ionization cooling" at the level required for a Higgs factory and eventually as premise for a subsequent multi-TeV collider and/or a long distance $v$ factory.

\section{Thank you !}

